Document downloaded from:

http://hdl.handle.net/10251/102618

This paper must be cited as:

Gamiz Gonzalez, MA.; Guldrís-Prada, P.; Antolinos Turpín, CM.; Ródenas Rochina, J.; Vidaurre, A.; Gómez Ribelles, JL. (2017). Fast degrading polymer networks based on carboxymethyl chitosan. Materials Today Communications. 10:54-66.

doi:10.1016/j.mtcomm.2017.01.005

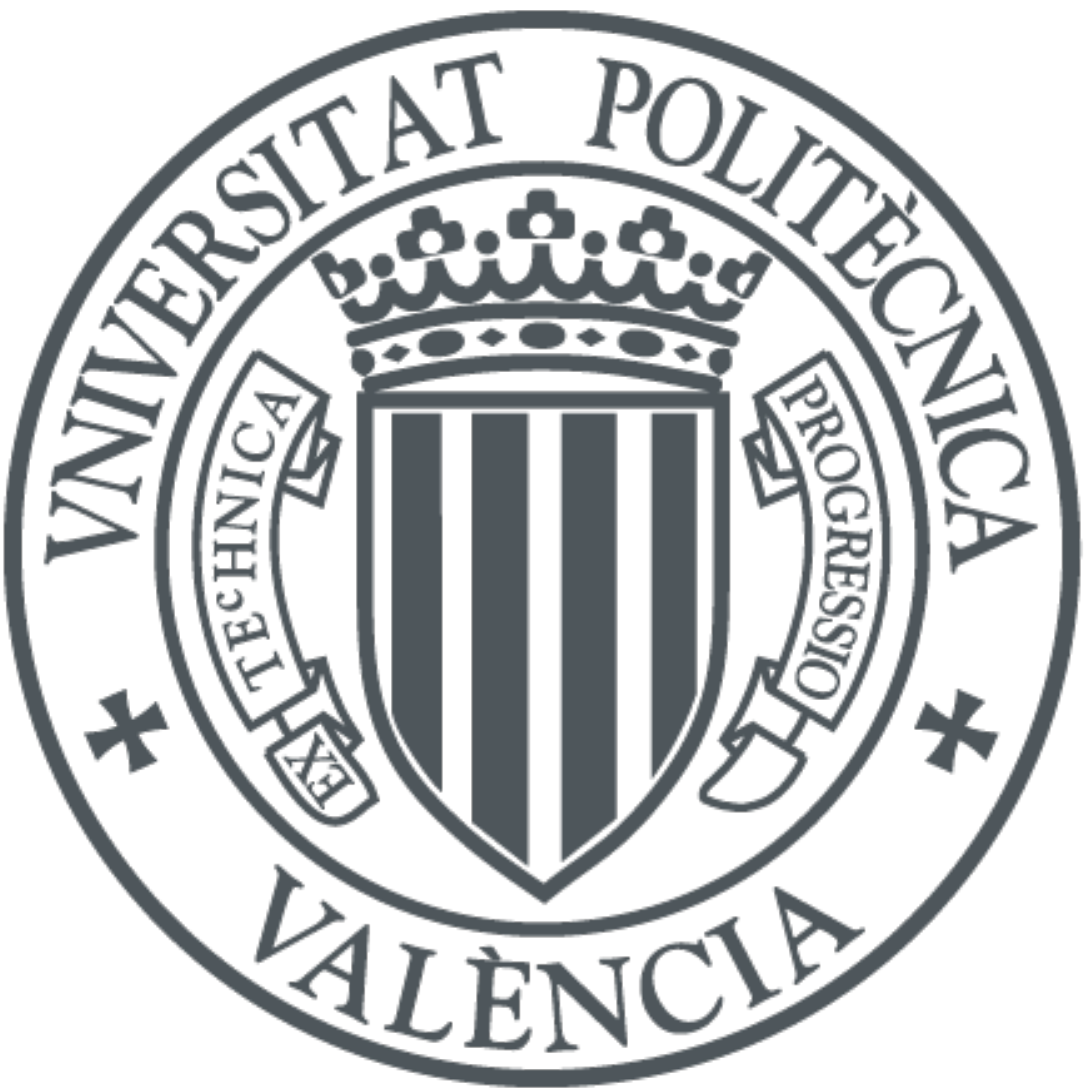

The final publication is available at

https://doi.org/10.1016/j.mtcomm.2017.01.005

Copyright Elsevier

Additional Information 


\title{
Fast degrading polymer networks based on Carboxymethyl Chitosan
}

\author{
M. A. Gámiz-González, ${ }^{a}$ P. Guldris, ${ }^{a}$ C. M. Antolinos Turpín ${ }^{\text {, J. }}$ \\ Ródenas Rochina $^{a}$, A. Vidaurre, ${ }^{\text {a,b }}$ J. L. Gómez Ribelles ${ }^{\text {a,b }}$
}

${ }^{\mathrm{a} C e n t e r ~ f o r ~ B i o m a t e r i a l s ~ a n d ~ T i s s u e ~ E n g i n e e r i n g ~(C B I T), ~ U n i v e r s i t a t ~ P o l i t e ̀ c n i c a ~ d e ~}$ València, Cno. de Vera s/n, 46022 Valencia, Spain.

${ }^{b}$ Networking Research Center on Bioengineering, Biomaterials and Nanomedicine (CIBER-BBN), Valencia, Spain

\begin{abstract}
In this work macroporous membrane for mesenchymal stem cells, MSCs, transplant has been developed. The membranes support cell seeding and proliferation and completely degrade in less than one week in "in vitro" culture. The biodegradable material is a polymer network based on carboxymethyl chitosan (a water soluble modification of chitosan) crosslinked by poly-(E-caprolactone) PCL, fragments which are susceptible to hydrolytic degradation. Synthesis was performed in solution in a common solvent for the two components of the network. The gel fraction was assessed by extraction in selective solvents. Physical characterization of networks of varying composition included water sorption capacity and the crystallinity of poly-( $\mathcal{E}$-caprolactone) in the network. In this way polymer networks are synthesized that loose between $66 \pm 5 \%$ and $89 \pm 1 \%$ of their mass when immersed in water for 28 days. The same weight loss is attained in enzymatic medium in only 4 days. Porcine bone marrow MSCs were seeded in macroporous membranes to show cell viability, and proliferation up to 7 days culture when the biomaterial is completely dissolved in the medium.
\end{abstract}




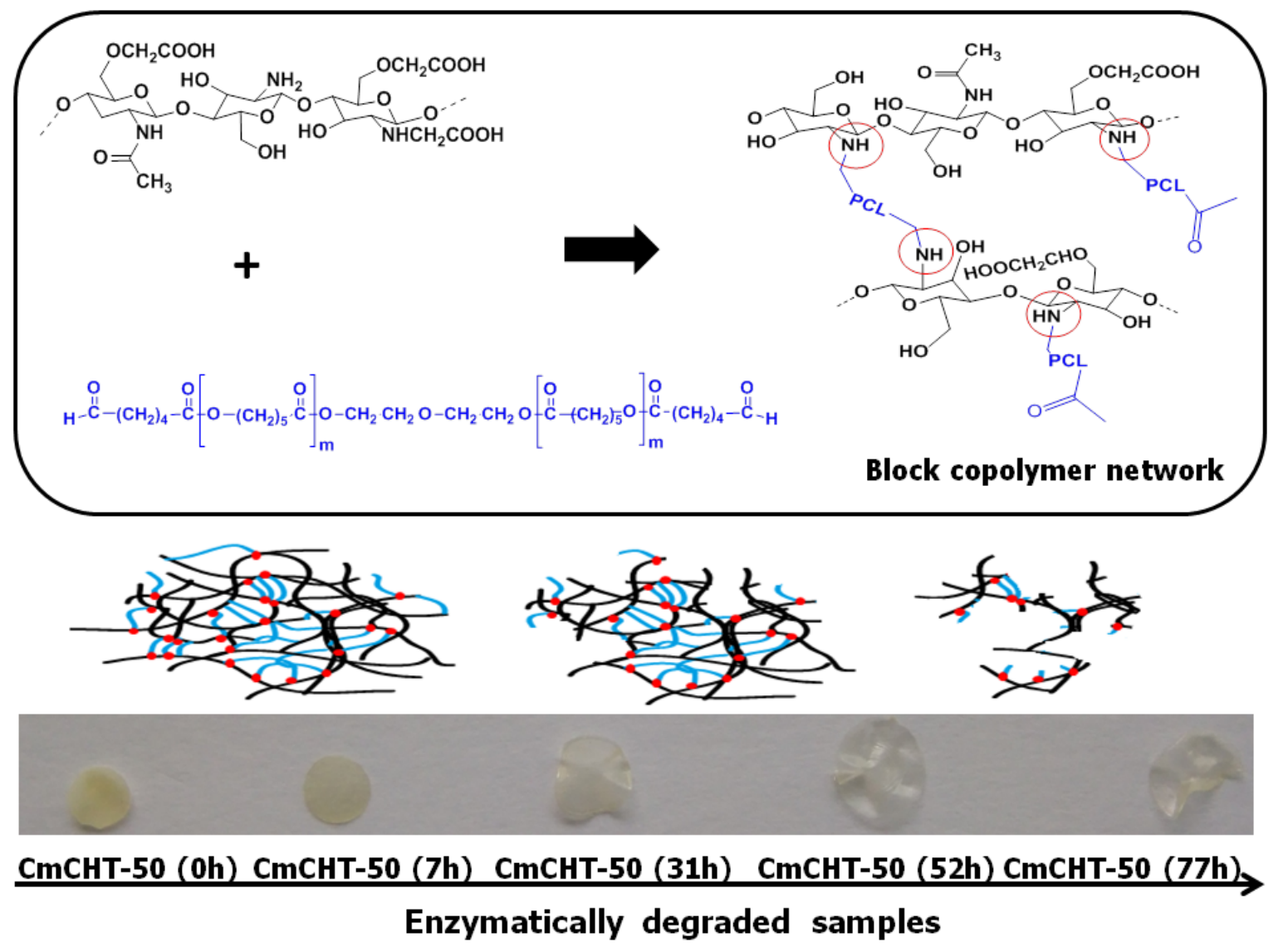

\section{Keywords}

Fast degradation, Mesenchymal stem cells, Carboxymethyl chitosan and poly ( $\mathcal{E}$ caprolactone) block-copolymer networks, Thermal stability.

\section{Introduction}

Chitosan (CHT) is a polysaccharide [1] used in a wide variety of tissue engineering applications such as bone, cartilage, and skin regeneration [2],[3],[4]. Chitosan is not watersoluble in a basic or neutral $\mathrm{pH}$ aqueous medium[5]. It is susceptible to enzymatic degradation by lysozyme [6]. Ren et al.[7] found that after 84 days of enzymatic degradation in lysozyme, the mass loss was only $10 \%$ for chitosan with a deacetylation degree of around $81 \%$. Hydrolytic degradation in aqueous media is nearly zero [8]. This limits chitosan applications in tissue engineering.

Carboxymethyl chitosan (CmCHT) is a water-soluble chitosan derivative. It is suitable as a biomaterial and is widely used in different fields such as tissue engineering applications, targeted drug delivery, and gene therapy. 
Chemical cross-linking of chitosan is the most common method for producing permanent polymer networks using covalent bonding between the polymer chains.[9] Genipin,[10] tripolyphosphate [11], and particularly dialdehyde crosslinkers such as glutaraldehyde,[12],[13],[14] have been used (among many others) as crosslinking agents. These copolymer networks have applications as supporting biomaterials in regenerative medicine therapies [15], [16] and for transplanting stem cells to organisms.

In this work, we used a new cross-linker based in a medium molecular weight poly-( $\varepsilon$ caprolactone) (PCL) chain. PCL is susceptible to hydrolysis although the hydrolytic degradation of bulk PCL is quite slow due to its hydrophobicity and high crystallinity[17]. PCL has been used in the production of scaffolds for tissue engineering applications, mainly in bone regeneration, cardiovascular and cartilage tissue engineering, Its low melting point (around $60^{\circ} \mathrm{C}$ ) allows easy modeling in a variety of shapes, even by 3D rapid prototyping and the low glass transition temperature $\left(-60^{\circ} \mathrm{C}\right)$ gives flexibility and impact resistance to the semicrystalline polymer [18]. Different combinations of chitosan and PCL have been proposed in the literature, in the form of blends or interpenetrated polymer networks [19], [20], [21].

In some regenerative therapies, the required resorption times of the biomaterial is quite short (in the order of few days) to encompass the degradation of the material to the growth of newly-formed tissue (for instance, in the case of skin regeneration or cornea treatments). The design of the biomaterial developed in this work is based on a block copolymer network formed of CmCHT chains, a water soluble polymer, crosslinked with PCL blocks (a hydrophobous, biodegradable polymer). The hypothesis was that the cleavage of PCL chains via hydrolytic or enzymatic degradation would deliver $\mathrm{CmCHT}$ chains that would simply dissolve in aqueous media. The crosslinking reaction was carried out between aldehyde end groups from PCL and amino groups from CmCHT in a Schiff's reaction[22]. Aldehyde end-caped poly-(E-caprolactone) (aPCL) was used as a crosslinker and synthesized using a Swern oxidation reaction [23] from PCL-diol. Polymer networks with different proportions of aPCL were synthesized. The physical-chemical properties of the synthesized polymer networks were analyzed using equilibrium water content, thermal degradation, and crystallinity. 
The degradation profiles in hydrolytic and enzymatic conditions (Lipase and Lysozime) were tested. The degradation was characterized by measuring the remaining weight, equilibrium water content, composition, and molecular weight of the degradation media. Pig mesenchymal stem cells, MSCs culture in the macroporous membranes, probes the viability, proliferation and fast degradation of the supporting biomaterial during "in vitro" cell culture.

\section{Experimental section}

\section{Materials}

Carboxymethyl chitosan (CmCHT) with a deacetylation degree (DD) of around $90 \%$ was obtained from Santa Cruz Biotechnology. The CmCHT was used as received without any purification. The substitution degree of carboxymethyl groups was analyzed with a potenciometric method described in the reference[24] and it is around DS=1.22 \pm 0 . carboxymethyl groups per monomeric unit. The molecular weight was measured [25] and it was around $\mathrm{MW}=677 \mathrm{kDa}$ with polidispersity index $\mathrm{P} Đ=2.64$. Poly ( $\varepsilon$-caprolactone) diol with a molecular weight (Mn) of $2000 \mathrm{Da}$, chloroform $\left(\mathrm{CHCl}_{3}\right)$, anhydrous dimethylsulfoxide (DMSO), oxalyl dichloride $\left(\mathrm{COCl}_{2}\right)$, diclromethane (DCM), trietylamine (TEA), magnesium sulfate $\left(\mathrm{MgSO}_{4}\right)$, cianoborohydryre sodic $\left(\mathrm{NaBH}_{3} \mathrm{CN}\right)$ and formic acid $(\mathrm{HCOOH})$, all analytical grade, were purchased from Sigma-Aldrich.

\section{Synthesis of poly (E-capolactone) dialdehyde (aPCL)}

All reaction vessels and reagents were manipulated in an inert atmosphere. Oxidation of PCL diol to obtain the PCL dialdehyde (aPCL) was carried out following Swern oxidation of Groth et al[23] using the DCM like a solvent The primary alcohols are oxidized to aldehyde groups in this reaction conditions. Oxalyl dichloride $(0.85 \mathrm{ml} ; 10 \mathrm{mmol})$ was dissolved in anhydrous DCM $(50 \mathrm{ml})$. The solution was cooled to $-47^{\circ} \mathrm{C}$. DMSO $(1.5 \mathrm{ml}$; $0.625 \mathrm{mmol}$ ), mixed with DCM, and slowly added to the oxalyl dichloride solution. PCL diol (5g; $2.5 \mathrm{mmol})$ previously dissolved in DCM (16 ml) was added to the cooled solution drop by drop. The mixture was stirred during 3 hours at $-47^{\circ} \mathrm{C}$. The catalytic amount of TEA (5.2 ml; $9.3 \mathrm{mmol}$ ) was then added and allowed to reach room temperature. The resulting product was diluted in DCM and extracted with $\mathrm{HCl} 1 \mathrm{M}$ and a supersaturated 
$\mathrm{NaOH}$ solution. The extracts were dried with $\mathrm{MgSO}_{4}$. The resulting solution was rotaevaporated and the final product was dissolved in chloroform and reprecipitated with cool diethyl ether and then dried in a vacuum.

\section{Synthesis of CmCHT-PCL network}

Carboxymethyl chitosan (CmCHT) and poly ( $\varepsilon$-caprolactone) dialdehyde (aPCL) were separately dissolved in formic acid (2\%( w/v)). Crosslinkig reaction took place at room temperature. Films with differing initial CmCHT:aPCL weight ratios: 95:5; 90:10; 80:20; 65:35; and 50:50 (w/w) were obtained after evaporation of the solvent in poly(tetrafluor ethylene) (PTFE) molds. The CmCHT-PCL films were carefully separated from the (PTFE) mold and treated with $\mathrm{NaBH}_{3} \mathrm{CN} 1 \%(\mathrm{w} / \mathrm{v})$ solution to reduce the $(\mathrm{C}=\mathrm{N})$ bond between aldehyde and amine groups from the aPCL and CmCHT respectively. The obtained polymer networks were washed with acetone and water to remove the not bonded polymer

chains from the cross-linked network and finally, the networks were dried for $48 \mathrm{~h}$ in a vacuum oven at $50^{\circ} \mathrm{C}$. The samples were named CmCHT-38, CmCHT-50, CmCHT-66, CmCHT-77 and CmCHT-93 where the number indicates the mass percentage of CmCHT calculated from thermogavimetric analysis (TGA).

\section{Proton nuclear magnetic resonance spectroscopy (1H-NMR)}

1H-NMR spectra were recorded at room temperature with a Varian 400 spectrometer (Varian, USA). The samples were dissolved in $\mathrm{CDCl}_{3}(\delta 7.28)$, and tetramethylsilane (TMS) was used as an internal reference.

\section{Fourier transforms infrared spectroscopy (FTIR)}

FTIR spectra of the samples were recorded on a Thermo Nicolet Nexus FTIR, using OMNUC software and the smart diffuse reflectance method. The samples were analyzed as a powder and the reference was dried $\mathrm{KBr}$. The spectra were obtained by continuously accumulating 32 scans in the $500-4000 \mathrm{~cm}^{-1}$ range at a $4 \mathrm{~cm}^{-1}$ resolution. 


\section{Solid State NMR spectroscopy (CP-MAS)}

Solid-state 13C NMR (CP-MAS) experiments were carried out on a Bruker Avance III Wide Bore $400 \mathrm{MHz}$ spectrometer at the resonance frequency of $100.6404 \mathrm{MHz}$ using 4 mm double resonance cross-polarization magic angle spinning probe.

Around $100 \mathrm{mg}$ of sample was packed into a $4 \mathrm{~mm}$ zirconium oxide rotor with a Kel-F endcap. 13C CP/MAS NMR spectrum was acquired with a cross-polarization contact time of 2 ms and a recycle delay of $5 \mathrm{~s}$ at a MAS speed of $10 \mathrm{kHz}$. All experiments were carried out at $300 \mathrm{~K}$ with high power proton decoupling during acquisition. The spectra were referenciated to $\alpha$-glycine as external reference.

\section{Water content}

Water content capacity of the polymer networks was assessed by immersion of the dry samples in deionized water. Samples ( $\mathrm{N}=3$ replicates) ranging between 1-2 mg were immersed in $3 \mathrm{ml}$ of deionized water. The weight of the samples was followed gravimetrically after careful elimination of the superficial water with a piece of filter paper. The equilibrium water content (EWC) was determined by Eq (1):

$$
E W C(\%)=\frac{m-m_{0}}{m_{0}} 100 \quad \text { Eq(1) }
$$

where $\mathrm{m}_{\mathrm{o}}$ refers to the initial weight in the dry state and $\mathrm{m}$ refers to the sample weight after $48 \mathrm{~h}$ of immersion, once equilibrium was reached. Weight was measured with a balance (Mettler Toledo) with a sensitivity of $0.01 \mathrm{mg}$.

The equilibrium water content, referred to the hydrophilic component weight, $\mathrm{m}_{\mathrm{CmCHT}}$, was determined by Eq (2).

$$
E W C^{\prime}(\%)=\frac{m-m_{0}}{m_{C m C H T}} 100
$$

\section{Thermogravimetryc analysis}

TGA measurements were performed using a TA-Instrument Model SDT-Q600 system. TGA tests were carried out in alumina crucibles where weight samples, between 5 and 10 mg, were heated from $30{ }^{\circ} \mathrm{C}$ to $900{ }^{\circ} \mathrm{C}$ at a heating rate of $10{ }^{\circ} \mathrm{C} / \mathrm{min}$. TGA experiments 
were performed using a nitrogen flow of $20 \mathrm{ml} / \mathrm{min}$ in order to avoid thermoxidative reduction.

The CmCHT proportion in the polymer network was determined by TGA from the residues obtained at $600^{\circ} \mathrm{C}$, RW600, excluding the water loss in the $R W_{180}$ samples (residue measured at $180^{\circ} \mathrm{C}$ ). Thus, the residue weight of the network, $R W_{\text {network, }}$ was calculated by applying the Eq (3):

$$
\mathrm{RW}_{\text {netwrok }}=\frac{\mathrm{RW}_{600}}{\mathrm{RW}_{180}} 100
$$

The mass fraction of CmCHT, $X_{C m C H T}$, and PCL blocks, $X_{a P C L},\left(\mathrm{X}_{\mathrm{CmCHT}}+\mathrm{X}_{\mathrm{aPCL}}=1\right)$ were calculated by applying the Eq (4),

$$
R W_{\text {network }}=\left(X_{C m C H T} R W_{C m C H T}+X_{a P C L} R W_{a P C L}\right) 100 \quad E q(4)
$$

Where $R W_{C m C H T}$ and $R W_{a P C L}$ are the CmCHT and aPCL residues obtained by applying Eq (3) to the pure components.

\section{X-ray diffraction}

X-ray diffraction spectra of the CmCHT-PCL polymer networks were obtained on a Rigaku Ultima IV X-ray diffractometer in the Bragg-Bentano configuration using the Ka radiation of a $\mathrm{Cu}$ anode. The networks were scanned from $2 \theta=5-70^{\circ}$ at a speed of $2^{\circ} / \mathrm{min}$. Diffraction patterns were deconvoluted using “PDXL” integrated X-ray powder diffraction software.

\section{Differential scanning calorimetry (DSC)}

Differential scanning calorimetry (DSC) was performed in a Pyris DSC8000 from TA Instruments, calibrated with indium and zinc standards in a nitrogen atmosphere. The dry samples (2-10 mg weight) were held at $30^{\circ} \mathrm{C}$ during 1 minute. They were then cooled from 30 to $-87^{\circ} \mathrm{C}$ at a cooling rate of $-20^{\circ} \mathrm{C} / \mathrm{min}$, held at $-87^{\circ} \mathrm{C}$ for $2 \mathrm{~min}$ and heated from -87 to $90{ }^{\circ} \mathrm{C}$ at a heating rate of $20^{\circ} \mathrm{C} / \mathrm{min}$, held at $90^{\circ} \mathrm{C}$ for $1 \mathrm{~min}$ and cooled and heated again under the same conditions. The final temperature was $30^{\circ} \mathrm{C}$. The degree of crystallinity of the PCL contained in the network was calculated, for both the first and second heating scan, from the area of the melting peak: 


$$
X_{P C L \text { Crist }}=\frac{\Delta H_{f}}{\Delta H_{P C L} W_{P C L}} 100
$$

Where $\Delta H_{f}$ is the heat of fusion of the polymer network, measured at the first or second heating scans, $\triangle H_{P C L}$ is the heat of fusion of the $100 \%$ crystalline PCL $139.5 \mathrm{~J} / \mathrm{g},[26]$ and $W_{P C L}$ is the PCL weight in the sample, as calculated from composition data.

\section{Enzymatic and hydrolytic degradation}

Samples with $5 \mathrm{~mm}$ of diameter ( $\mathrm{N}=5$ replicates at each degradation time) were degraded under hydrolytic or enzymatic conditions. Samples were immersed in a PBS solution to check the hydrolytic degradation $(\mathrm{pH}=7.4$ and $0.02 \%(\mathrm{w} / \mathrm{v})$ of sodium azide to avoid bacteria proliferation). The enzymatic degradation media was prepared adding $1 \mathrm{mg} / \mathrm{ml}$ of lysozyme or lipase to PBS $(\mathrm{pH}=7.4)$ with $0.02 \%$ (w/v) of sodium azide[27]. The temperature was held at $37{ }^{\circ} \mathrm{C}$ during the degradation experiments, and the degradation media was replaced once a week in the hydrolytic degradation and twice a week in the enzymatic degradation. At predefined degradation intervals, samples were extracted from the degradation media, carefully dried with a piece of filter paper and weighed to determine the equilibrium water content by Eq (1). Samples were placed in vacuum desiccators at room temperature until a constant weight was reached. The percentage of the remaining weight after degradation was determined by comparison of the weight of the dry sample before the degradation process $\left(m_{o}\right)$, with the weight of the dry sample, $m_{d}$, using the following equation $\mathrm{Eq}(6)$ :

$$
\begin{aligned}
& \text { Remaining weight }(\%)=\frac{m_{d}}{m_{o}} 100 \quad E q(6)
\end{aligned}
$$

\section{Gel permeation chromatography}

Molecular weight distribution of supernatant solutions from enzymatic degradation media were analyzed using a Gel Permeation Chromatographer (GPC), at $35{ }^{\circ} \mathrm{C}$. Measurements were performed using a Waters Breeze GPC system with a 1525 Binary HPLC pump (Waters Corporation, Milford, MA) equipped with a 2414 refractive index detector and four serial columns of water (Ultrahydrogel $7.8 \mathrm{~mm}$ ID x $30 \mathrm{~cm}$ ). The supernatant solutions were lyophilized to remove the water and obtain a concentrate product, and dissolved in 
3ml of mili Q water. PBS solution ( $\mathrm{pH}=7.5-7.4)$ was used as mobile phase at a flow rate of $0.5 \mathrm{ml} / \mathrm{min}$ and $100 \mu \mathrm{l}$ injection volume. The calibration curve was prepared using monodisperse PEO (polyethylene glycol) standard kits (Waters).

\section{Cell isolation and sub culture}

Mesenchymal stem cells (MSCs) were collected from femora bone marrow obtained from a 4-month-old porcine specimen. Under laminar flow hood femora head was removed and gelatinous bone marrow was collected from medullary cavity. Bone marrow femora was resuspended and homogenized in DMEM GlutamaX supplemented with 10\% FBS and 1\% penicillin/streptomycin. Cell suspension was centrifuged at $650 \mathrm{~g}$ for $5 \mathrm{~min}$ and supernatant discarded, and repeating once again after going back to resuspended cells. The resulting pellet was again resuspended in culture medium and filtered using a $40 \mu \mathrm{m}$ nylon filter. Cells were seeded in a T75 $\mathrm{cm}^{2}$ culture flask at 4 x 105 cells/cm2 and expanded in monolayer culture until passage 1 with Dulbecco's Modified Eagle's Medium, DMEM GlutamaX (Gibco, UK), supplemented with 10\% of fetal bovine serum, FBS (Gibco, UK), 2\% penicillin/streptomycin (P/S) (Gibco, UK), $5 \mathrm{ng} / \mathrm{mL}$ human recombinant fibroblast growth factor-2 (FGF-2) (Eurobio) and $125 \mathrm{mg} / \mathrm{mL}$ amphotericin B (Sigma Aldrich, Spain). Expansion step was repeated twice more until passage 3. Cells were concentrated (at 1.5x106 cells $/ \mathrm{mL}$ ) and cryopreserved in freezing medium (90\% FBS with 10\% dimethyl sulfoxide). Previous to cell seeding passage 4 was started, cells were unfrozen at $37^{\circ} \mathrm{C}$ in a water bath for $1 \mathrm{~min}$ and seeded at 5x103 cells/cm2 in a T75 cm2 culture flask.

\section{Sample disinfection and membranes cell seeding}

Cell culture after undergoing biological degradation (3h, 1, 3 and 6 days from seeding) was assessed in the porous membranes of carboxymethyl chitosan ( $\mathrm{CmCHT}$ ) and poly ( $\varepsilon$ caprolactone), CmCHT-77 and CmCHT-50, respectively, with porcine bone marrow MSCs.

Passage 4 cells expanded with DMEM supplemented with 5 ng/mL FGF-2 were detached using trypsine and suspended in free serum DMEM GlutamaX enriched, supplemented with $1 \% \mathrm{P} / \mathrm{S}$ to obtain a final concentration of $5 \times 106$ cells $/ \mathrm{ml}$. The membranes were cut in circular discs of $6 \mathrm{~mm}$ in diameter and then were disinfected with fungicides and broad- 
spectrum antibiotics treatment (several washes with deionized sterile water, $100 \square \mathrm{g} / \mathrm{mL}$ of fungizone antimycotic ( Fisher, Spain) and $50 \square \mathrm{g} / \mathrm{mL}$ of ampicilline (Sigma Aldrich, Spain) dissolution. The samples were frozen at $-80^{\circ} \mathrm{C}$ and lyophilized under sterile conditions, obtaining the dried samples prior to cell culture.

Cell seeding into the all samples was performed pippeting $20 \mu \mathrm{L} / \mathrm{sample}$ at $5 \times 106 \mathrm{cells} / \mathrm{ml}$ on the surface of each membrane and placing them in the incubator $30 \mathrm{~min}$ at $37^{\circ} \mathrm{C}$ in a $5 \%$ CO2 atmosphere. Afterward, $1500 \mu \mathrm{L} /$ sample of medium DEMEN GlutamaX enriched with $10 \% \mathrm{FBS}, 1 \% \mathrm{P} / \mathrm{S}$ and $5 \mathrm{ng} / \mathrm{mL}$ FGF-2 was deposited in samples and cultured at $37^{\circ} \mathrm{C}$ in normoxic conditions. Culture medium was changed four days after cell seeding and samples were collected at 3h, 1, 3 and 6 days.

\section{Indirect cytotoxicity assay}

Sample cytotoxicity was assed using protocols adapted from the ISO 10993-5 normative. Samples were disinfected following the antibiotic treatment described previously, as negative control was selected polystyrene and as positive control latex that were both sterilized with steam. Samples were weighed and cut following the guidelines of ISO 10993-12 previous to immerse in supplemented DMEM without phenol red for 24 hours at $37^{\circ} \mathrm{C}$. Cytotoxicity was assessed with L-929 murine fibroblasts that were expanded with DMEM supplemented with $10 \%$ of FBS and 1\% of P/S and seeded at 10000 cells/well in a 96 well plate. Material liquid extract was added 24 hours after cell seeding. Cell viability was measured 24 hours after initiate the non-contact assay. MTT solution at $10 \%$ in DMEM media without phenol red was added and incubated 3 hours at $37^{\circ} \mathrm{C}$. MTT solution was removed and precipitated salts were dissolved in Nonidet at $0.1 \%$ in isopropanol with $4 \mathrm{mM}$ of $\mathrm{HCl}$. Well plate was read at $570 \mathrm{~nm}$.

\section{DNA Quantification biochemical assay}

Sample’s DNA content was determined using P7589 Quant-iT Picogreen dsDNA assay kit (Invitrogen). Four samples for each type of membrane were removed at 3h, 1, 3 and 6 days. Samples were washed with/in Dulbecco's phosphate buffered saline, DPBS, and stored in a/an eppendorfs at $-80^{\circ} \mathrm{C}$ previous to samples digestion. Samples were digested with proteinase K to release the DNA that was measured with Quant-iTTMPicoGreen ${ }^{\circledR}$ dsDNA Kit following the protocol described in previous works [28], [29], [30]. 


\section{Tridimensional cell distribution}

Tridimensional cell nucleus distribution was determined by fluorescence with DAPI nuclear staining using a confocal fluorescence microscope mounted on an inverted Zeiss microscope and software Nis-elements (Nikon). Samples were permeabilized with Triton X-100 0.1\% and incubated with DAPI solution 20 min previous to mount the samples with Fluorsave Vectashield mounting medium (ATOM) to stain the nucleus. Pictures were taken in 4 randomly selected sample's regions to obtain 3D (Tridimensional) representation of nuclei distribution and cell penetration/colonization depth value.

\section{Statistical analysis}

Statistical analysis of the data was performed using SPSS software. Differences between the groups with $\mathrm{p}<0.05$ were considered statistically significant.

Statistical analysis of cell quantification assay was done by two-way ANOVA for multiple comparisons using Graph-Pad Prism software. In figures, the bars indicate the standard error and asterisk indicate statistically significant differences. In this case statistical significance was laid on $\mathrm{p}<0.05$.Results and discussions

\section{Results and Discusion}

\section{Synthesis of Poly (E-capolactone) dialdehyde (aPCL)}

The final product was characterized by $1 \mathrm{H}-\mathrm{NMR}$ spectroscopy by comparing the spectrum of PCl diol (Figure 1a) with the corresponding aPCL (Figure 1b). Both exhibited identical signals corresponding to the polymer backbone with the exception of two new peaks that appear at around 2.5 and $9.5 \mathrm{ppm}$. These signals are attributed to the methylene neighbor to the aldehyde group (i) and the proton from the aldehyde group (j). Another characteristic change is observed in the signal of the $\left(\mathrm{CH}_{2}\right)$ h proton neighbor to the alcohol group, which decreases in the aPCL product due to the substitution of the original alcohol by aldehyde end-capped. 


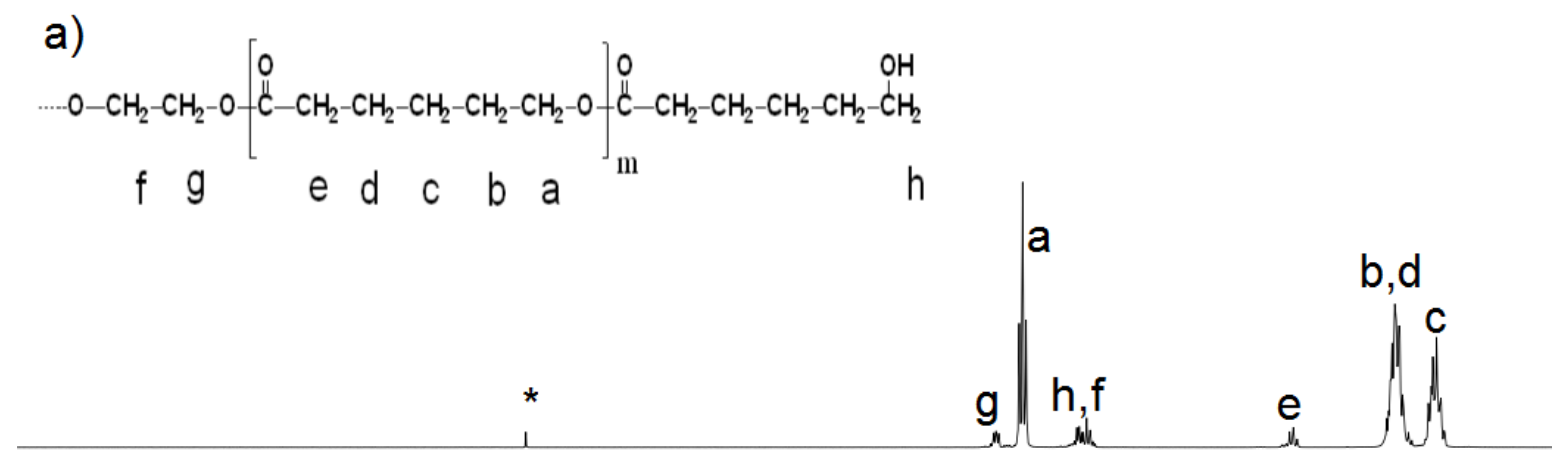

b)

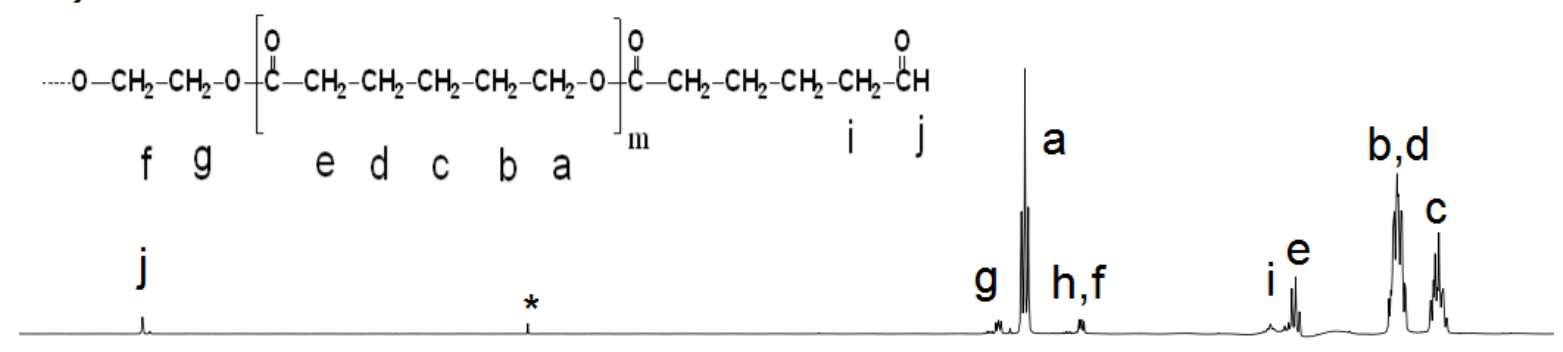

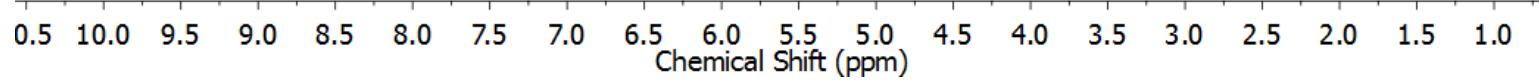

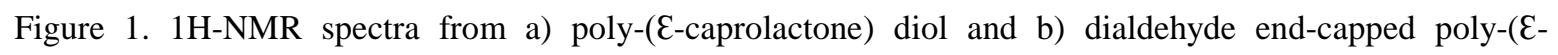
caprolactone) (aPCL) polymer.

The conversion degree (DC) of the aldehyde end-capped polymer (aPCL) was evaluated from the integral of the aldehyde group ( $\mathrm{j}$ ) at $9.78 \mathrm{ppm}$ and taking as a reference the area of the $-\mathrm{CH}_{2}$ (c)- appearing at $1.40 \mathrm{ppm}$, which is common to the starting PCL diol and the modified aPCL. Thus, a value of conversion degree (DC) of approximately $40 \pm 18 \%$ was found. This result is agree with the results reported by Groth et al [23], where only modest degrees of oxidation between 40 and $60 \%$ were obtained presumably due to the polymer precipitation during the reaction process. Being this conditions the reaction the high yield of the oxidation, comparing with the others oxidation conditions tested by the author.

\section{CmCHT crosslinking with aPCL}

The CmCHT-PCL polymer networks were obtained by dissolving the desired proportions of aPCL and CmCHT in formic acid at room temperature. A covalent bonding between aldehyde group from aPCL and the amine group from CmCHT, forming a cross-linked 
network is expected. This is a typical reaction of chitosan resulting in Schiff's base formation [22]. The obtained imines $(-\mathrm{N}=\mathrm{C}-)$ were reduced with $\mathrm{NaBH}_{3} \mathrm{CN}$ to obtain a robust crosslinking amine group (-N-C-).

Cross-linking of CmCHT by aPCL is proved by solvent extraction experiments. The nonbonded polymer chains were extracted after network reduction by washing the samples several times with acetone (good solvent for PCL chains) and water (good solvent for CmCHT). The remaining weight after reduction and washing was measured gravimetrically (3 replicates) (see Table 1). The yield of the polymer network formation was around $70 \pm 3 \%$.

Table 1. Remaining weight after: a) $\mathrm{NaBH}_{3} \mathrm{CN}$ (reduction) and washes with b) water and c) acetone.

\begin{tabular}{llll} 
Sample code & $\begin{array}{l}\text { Remaining } \\
\text { weight } \\
\text { reduction (\%) }\end{array}$ & $\begin{array}{l}\text { Remaining weight } \\
\text { after washing in } \\
\text { water (\%) }\end{array}$ & $\begin{array}{l}\text { Remaining weight } \\
\text { after washing in } \\
\text { acetone (\%) }\end{array}$ \\
\hline CmCHT-77 & $72 \pm 2$ & $73 \pm 1$ & $71 \pm 3$ \\
CmCHT-66 & $77 \pm 2$ & $75 \pm 4$ & $73 \pm 1$ \\
CmCHT-50 & $79 \pm 4$ & $74 \pm 5$ & $71 \pm 1$ \\
CmCHT-38 & $84 \pm 5$ & $69 \pm 4$ & $66 \pm 7$ \\
\hline
\end{tabular}

Extraction of the unreacted CmCHT starts in the reduction step, in which between 16 and $28 \%$ of the initial mass of CmCHT and aPCL is extracted depending of the sample composition. After reduction, further extraction of $\mathrm{CmCHT}$ with washing in water is not significant. On the other hand and the amount of PCL extracted (referring to the initial mass of aPCL) when washing with acetone ranges from $3 \pm 7 \%$ for $\mathrm{CmCHT}-77$ to $15 \pm 4 \%$ for CmCHT-38.

\section{Composition and effect of crosslinking on thermal degradation}

The fraction of CmCHT was determined from the residue in TGA analysis. This fraction is in good agreement with what was expected from the initial amounts of reactants and the amounts extracted with solvent washing. (Figure 2). For the CmCHT polymer, the thermal degradation occurs in two steps; the first at around $180^{\circ} \mathrm{C}$ is attributed to water loss and represents around 5\% of the initial weight.[31] The second step, in the DTG curve, can be 
observed in a narrow peak between $200{ }^{\circ} \mathrm{C}$ and $320^{\circ} \mathrm{C}$, and is due to polymer thermal degradation, followed by a constant mass loss from $320^{\circ} \mathrm{C}$ and higher temperatures - and with a residual weight of around $45 \%$ at $600{ }^{\circ} \mathrm{C}$. The aPCL polymer presents a single thermal degradation peak between $300^{\circ} \mathrm{C}-500^{\circ} \mathrm{C}$, and its thermal degradation presented a 3 wt $\%$ of residue at $600^{\circ} \mathrm{C}$.

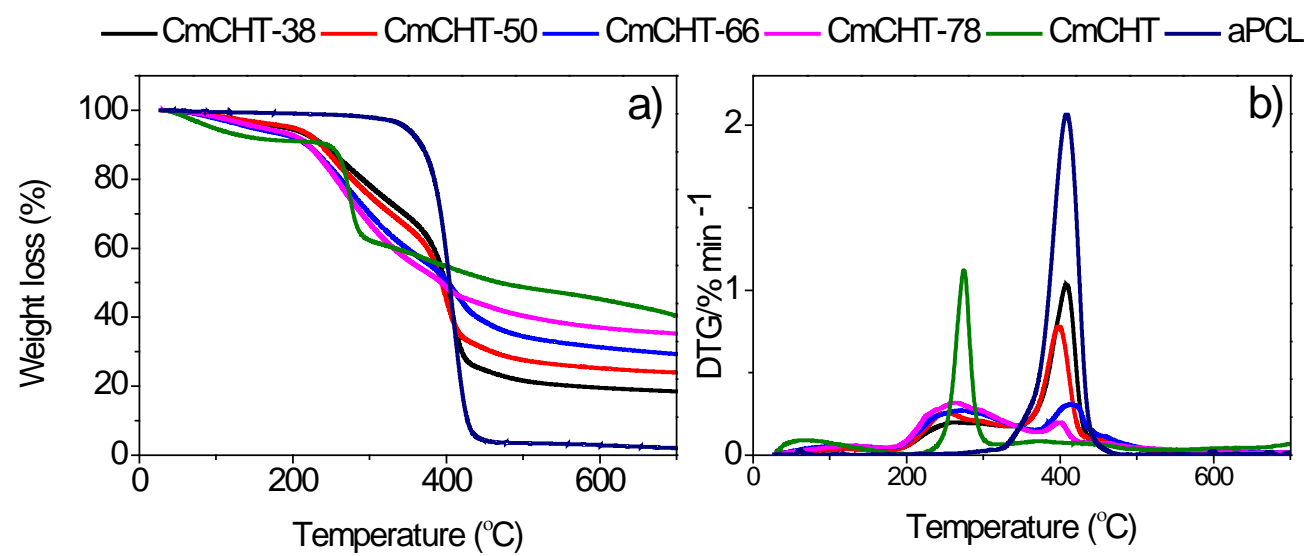

Figure 2. a)TGA and b) DTG curves of the networks with different compositions and starting polymers.

The weight loss of the polymer networks exhibited a complex profile between $200^{\circ} \mathrm{C}$ and $500^{\circ} \mathrm{C}$ and showing the main features of the pure components and some differences. Whereas the peak corresponding to PCL thermal degradation maintains its position and decreases in intensity when PCL content decreases, the peak corresponding to CmCHT thermal degradation broadens greatly with small differences among differently composed networks (Fig 2b). The broadening of the temperature interval in which the main degradation of the chitosan chains takes place indicates that an important amount of CmCHT monomeric units is interacting with PCL chains. As expected, when PCL content increases, the polymer network showed less thermal stability and also presented less residue weight than the pristine CmCHT polymer.[32] Although all polymer networks were dried in a vacuum before the TGA experiment, there was a weight loss in the interval from $30^{\circ} \mathrm{C}$ to $180^{\circ} \mathrm{C}$ due to the presence of some residual water (5-9 wt\%).

Table 2. Weight residue calculated at $180{ }^{\circ} \mathrm{C}$ and $600{ }^{\circ} \mathrm{C}$. Experimental CmCHT proportion obtained from Eq (4). 


\begin{tabular}{|c|c|c|c|c|}
\hline Sample code & $\begin{array}{l}\text { Remaining } \\
\text { weight } \\
\text { measured at } \\
180^{\circ} \mathrm{C}(\% \pm 1 \%)\end{array}$ & $\begin{array}{l}\text { Residue } \\
\text { measured at } \\
600^{\circ} \mathrm{C}(\% \pm 1 \%)\end{array}$ & $\begin{array}{l}\text { Initial CmCHT } \\
\text { proportion (\%) }\end{array}$ & $\begin{array}{l}\text { Measured } \\
\text { CmCHT } \\
\text { proportion } \\
(\% \pm 2 \%)\end{array}$ \\
\hline CmCHT & 91 & 45 & & \\
\hline CmCHT-93 & 90 & 32 & 90 & 93 \\
\hline CmCHT-77 & 90 & 35 & 95 & 77 \\
\hline CmCHT-66 & 93 & 31 & 80 & 66 \\
\hline CmCHT-50 & 96 & 25 & 65 & 50 \\
\hline CmCHT-38 & 95 & 20 & 50 & 38 \\
\hline aPCL & 99 & 3 & & \\
\hline
\end{tabular}

The weight fraction of CmCHT in the network was lower than the initial proportion, except for CmCHT-93. This means that most of the aPCL was crosslinked as can be seen in the composition calculated by TGA.

TGA thermograms also give information on the close interaction between the two components of the network. The thermal degradation of CmCHT presents a narrow peak in the weight derivative and shows a wide process in the networks with an approximate temperature interval between 180 and $380^{\circ} \mathrm{C}$. This may be related to the new covalent bonding between CmCHT amines and aldehyde end groups of aPCL.

\section{FTIR spectra}

The structural composition of the polymer networks was confirmed by analyzing the FTIR spectra (Figure 3). It shows the characteristics peaks of CmCHT at $3400 \mathrm{~cm}^{-1}$ (-OH stretch) and $2874 \mathrm{~cm}^{-1}$ (-CH- stretching bond). The bands at 1613-1426 $\mathrm{cm}^{-1}$ correspond to the carbonyl group $(v-\mathrm{C}=\mathrm{O})$ from amide I and carboxylic acid, respectively, and the band at $1312 \mathrm{~cm}-1$ corresponds to C-N stretching, amide II. The intense overlapped peaks from 1200-900 cm-1 correspond to several bond vibrations (such as C-O-C, C-O, C-O-H stretching, $\mathrm{N}-\mathrm{H}$ bending and $\mathrm{OH}$ and $\mathrm{C}=\mathrm{O}$ deformation). The aPCL component (spectrum $\mathrm{E}$ in Figure 3) shows a band at $1725 \mathrm{~cm}^{-1}$ due to the $\mathrm{C}=\mathrm{O}$ of the ester group, and the characteristic bands of -CH2- alkyl group that appears between 2952 and2856 $\mathrm{cm}^{-1}$. 
As the PCL content in the polymer networks increased, the intensity of its characteristic peaks at 1725 and 2952-2856 $\mathrm{cm}^{-1}$ increased. The broad band observed between $3600 \mathrm{~cm}^{-1}$ and $3000 \mathrm{~cm}^{-1}$ due to the inter and intra molecular hydrogen bonds of the $\mathrm{NH} 2$ and $\mathrm{OH}$ groups of the CmCHT decreases as the PCL content in the network increases. The peaks from amide I and amide II in the polymer networks presented lower intensity than CmCHT still difficult to characterize for CmCHT-50 and CmCHT-38. For CmCHT-77 (spectra B), the amide I and II shifted towards a higher wave number with respect CmCHT- indicating the crosslinking between CmCHT and PCL.

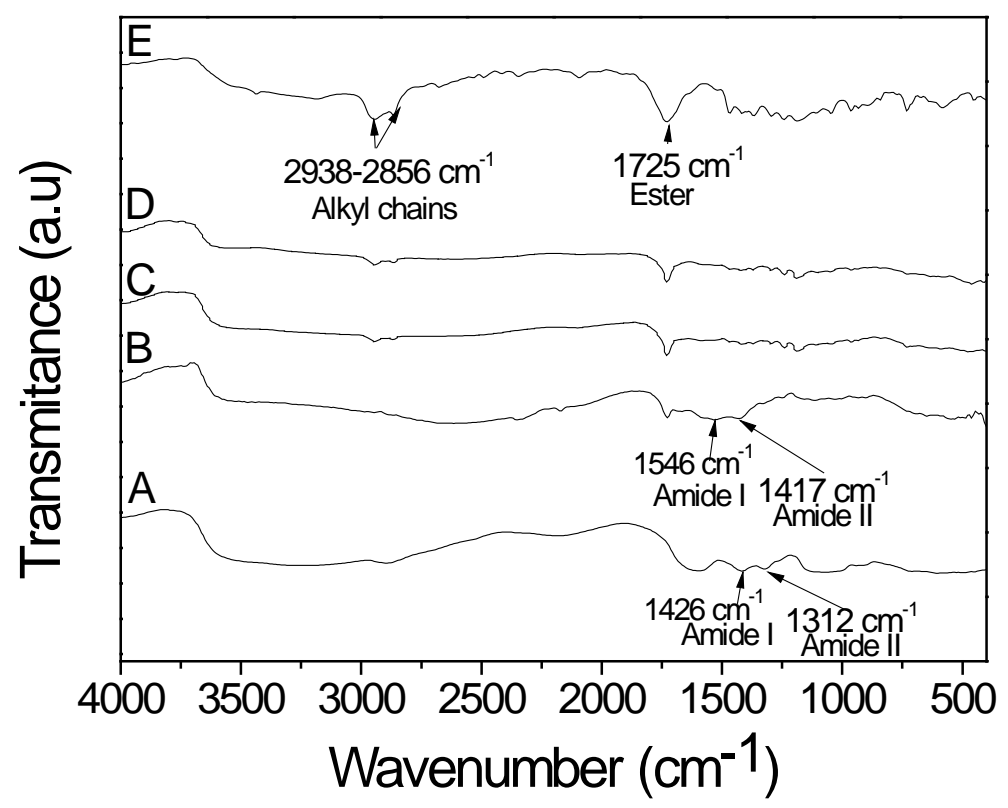

Figure 3. FTIR spectra of A) CmCHT, B) CmCHT-77, C) CmCHT-50, D) CmCHT-38 and E) aPCL.

\section{Solid State NMR spectroscopy (CP-MAS)}

The crosslinking between aPCL and CmCHT was analyzed by 13C solid state NMR spectroscopy. 

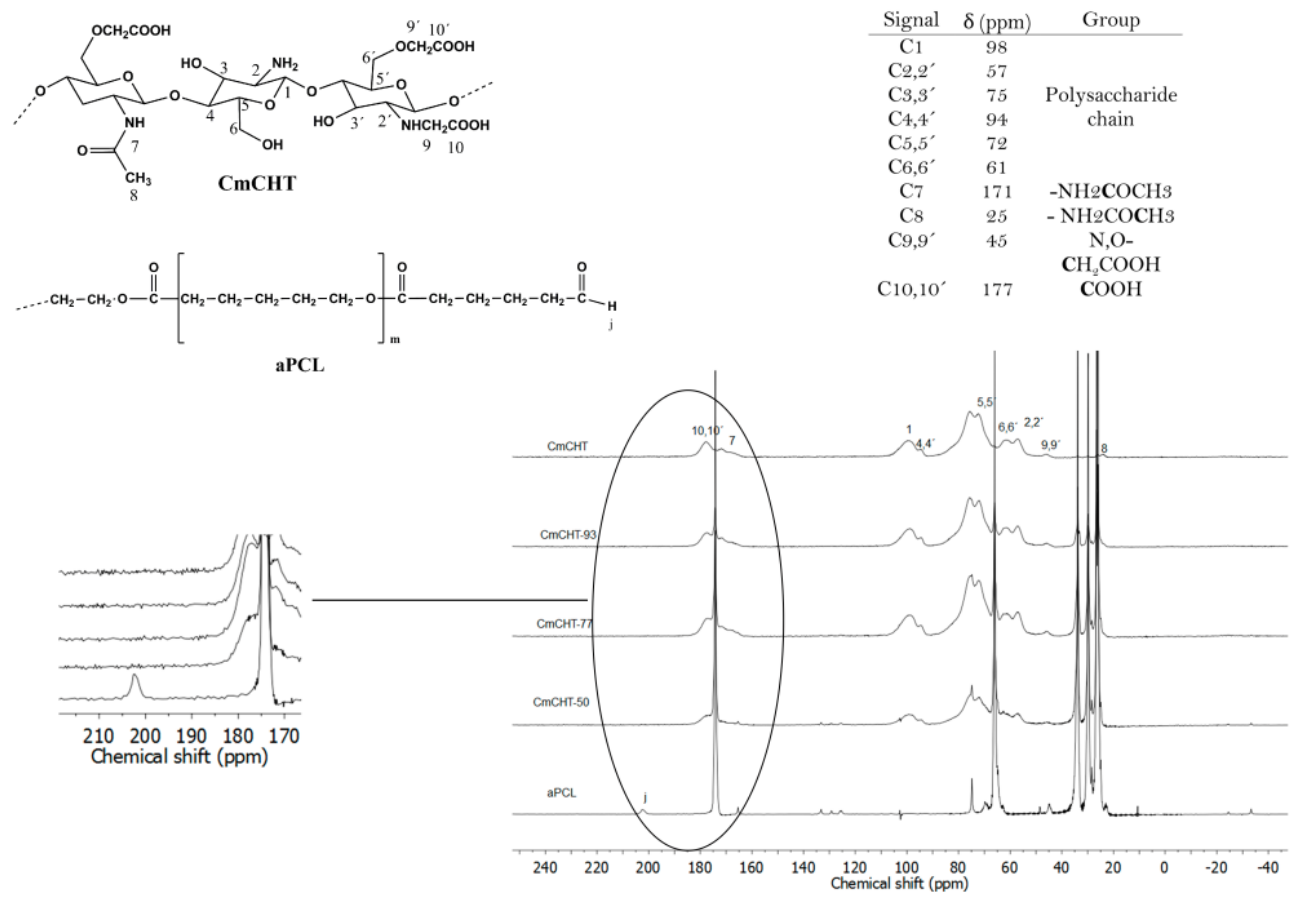

Figure 4. 13C solid state NMR spectra of carboxymethyl chitosan, dialdehyde end-capped poly(Ecaprolactone) and their graft copolymers.

Figure 4 shows the spectra from pure aPCL and CmCHT polymers and the CmCHT-50, CmCHT-77 and CmCHT-93 samples, in which each peak was identified following the literature for CmCHT polymers. The corresponding signals from each polymer appeared in all 13C solid state NMR spectra at intensities that varied with the proportion of polymer in the network.

For the crosslinking reaction in which the (-C-N-) linkage was activated: in the $13 \mathrm{C}$ solid state NMR spectra the signal corresponding to (-C-N-) linkage was difficult to characterize but the aldehyde group signal that appeared at 202 ppm in aPCL dissapeared in all 13CNMR spectra of the CmCHT-50, CmCHT-77 and CmCHT-93 networks, indicaiting the effective crosslinking between the polymers.

\section{Equilibrium water content}

The equilibrium water content, EWC, of the polymer networks obtained by Eq.1 is shown in Figure 5 (black bars). All the obtained polymer networks are hydrogels due to their capacity to host large amounts of water without being solubilized. CmCHT-77 presents a EWC $260 \pm 25 \%$. Despite the PCL content increases in the polymer network, the water absorption capacity does not decrease; this indicates that the PCL is forming disperse 
agglomerates in the network that do not hinder the swelling of the hydrophilic phase. Statistical analysis indicates that there are significant differences between the EWC of CmCHT-77 and the rest of the samples, but there are no significant differences between the EWC of samples CmCHT-66, CmCHT-50, and CmCHT-38.

Figure 5, also shows the amount of water referred to the content of hydrophilic component (grey bars), as it is obtained from Eq. 2. Except for the case of CmCHT-38, statistical analysis shows no significant change in EWC'. This fact suggests phase separation being the water molecules mainly accommodated in the hydrophilic domains that maintain a similar capacity for water absorption. However, water absorption is significantly lower than that of CmCHT-38 given that it has less hydrophilic component. This effect has been found in other hydrophobic/hydrophilic networks[33]'[34] and has been linked with the presence of favored intermolecular hydrogen bonds between polymer chains when there is a larger amount of hydrophilic component, leading to less available sorption sites for water molecules[35].

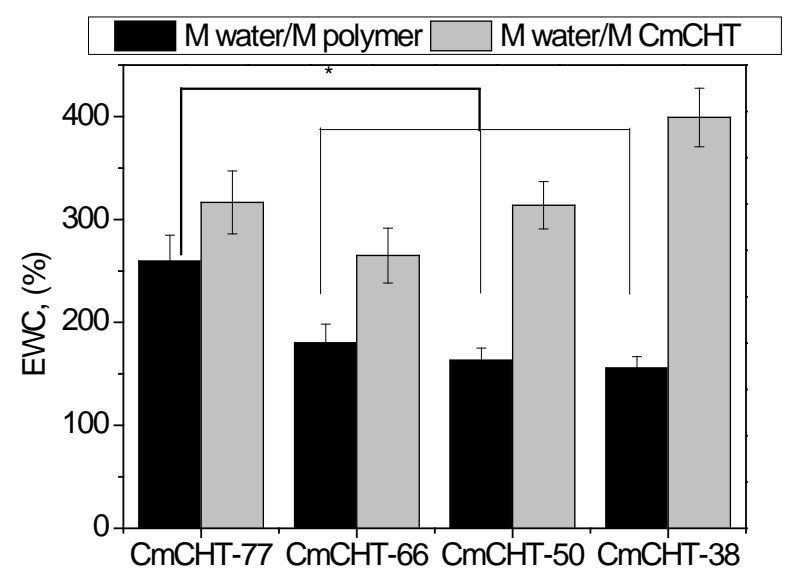

Figure 5. Equilibrium water content of the CmCHT-PCL polymer network - referred to the mass of polymer (black) and to the mass of hydrophilic component (grey).

\section{Miscibility and phase behavior analyzed by X-ray diffraction and DSC}

Figure 6 shows the DRX diffractograms of the polymer networks, as well as the pure CmCHT. Diffraction peaks were deconvoluted using non-symmetrical Gaussian peaks (shown in grey in Figure. 6). The diffraction pattern of CmCHT showed two broad and poorly defined peaks around $2 \Theta \approx 13^{\circ}$ and $2 \Theta \approx 25^{\circ}$. According to the literature,[36],[37] the 
CmCHT has a less ordered structure than CHT and presents two intense peaks around $2 \Theta \approx 10^{\circ}$ and $2 \Theta \approx 20^{\circ}$. The substitution of the carboxymethyl group breaks the hydrogen bonds between polymer chains and thus decreasing crystallinity. All the polymer networks showed the characteristic diffraction pattern of PCL with peaks at $2 \Theta \approx 21.3^{\circ}$ and $23.7^{\circ}$, which correspond to (110) and (200) crystallographic planes, respectively.[38] It is worth noting that even CmCHT-93 with only 7\% of PCL presented the characteristic PCL peaks that became better defined as the PCL proportion increased. These results indicate that the PCL domains are large enough to organize the polymer chain in crystalline structures.

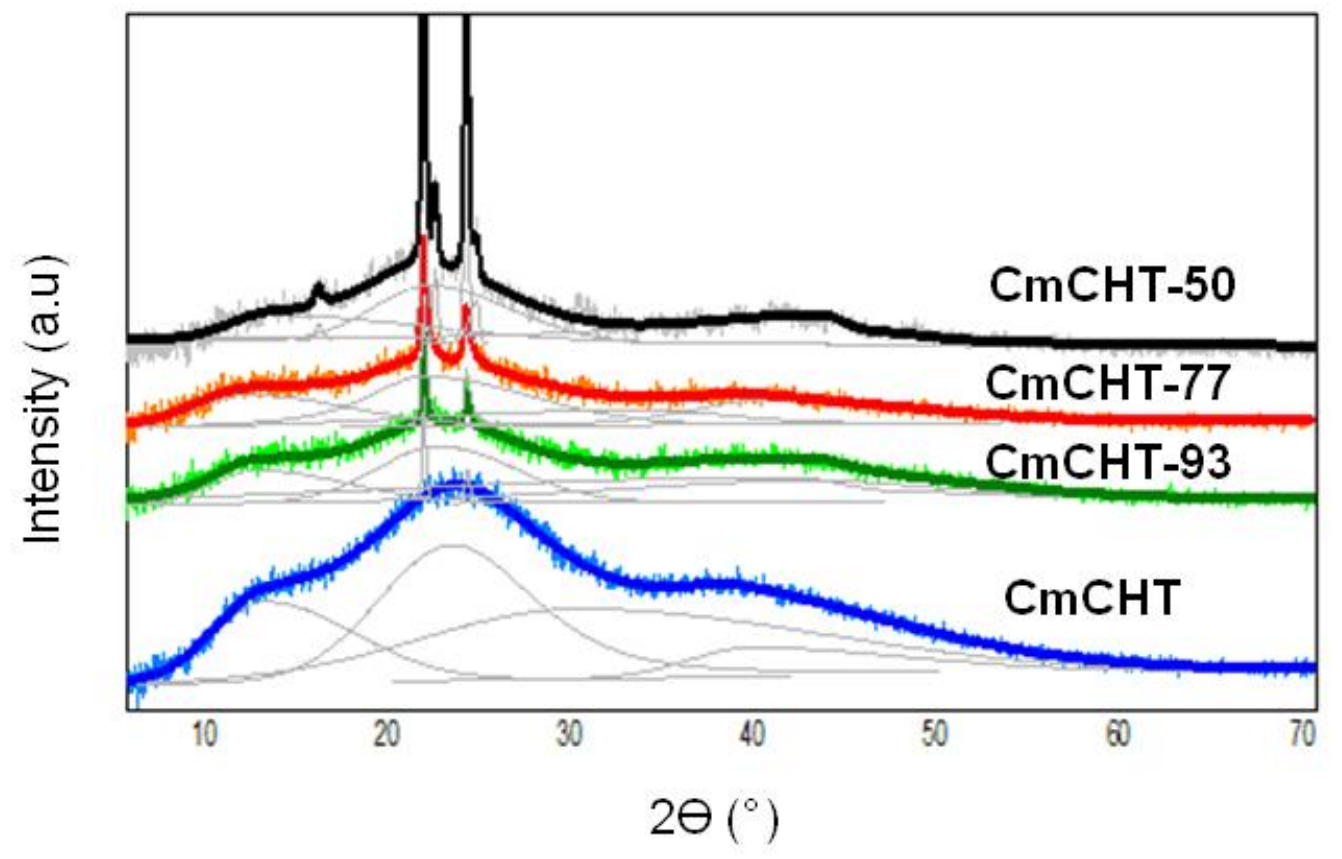

Figure 6. Diffractograms of CmCHT-PCL polymer networks.

Thermal properties were evaluated, by means of DSC experiments following the aforementioned conditions. In Figure 7, the second heating scan is represented for all polymer networks and also for pristine polymers. 


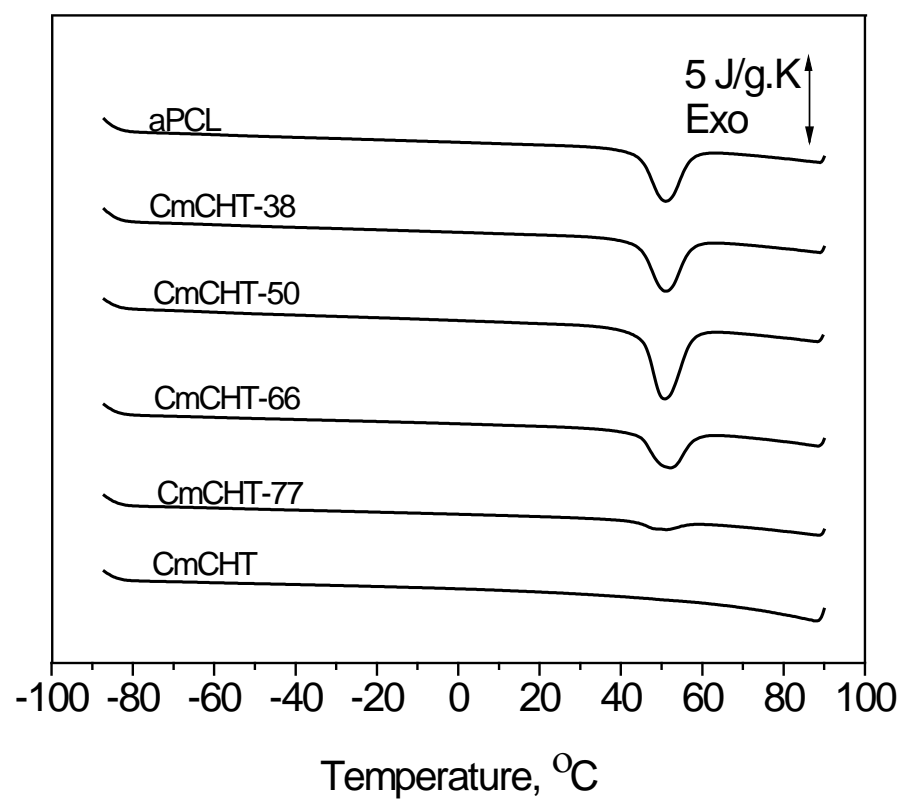

Figure 7. DSC thermograms corresponding to the second heating scan at a heating rate of $20^{\circ} \mathrm{C} / \mathrm{min}$ for the polymers networks - as well as CmCHT and aPCL.

The glass transition temperature $(\mathrm{Tg})$ for the aPCL is around $-60^{\circ} \mathrm{C}$. The aPCl polymer shows a melting temperature of $\mathrm{Tm} \approx 51^{\circ} \mathrm{C}$. However, the $\mathrm{CmCHT}$ did not show any characteristic glass transition temperature or melting peak in DSC spectra[39]. All the polymer networks presented the characteristic melting peak corresponding to PCL (around $51^{\circ} \mathrm{C}$ ). The area of the endotherm increased when the PCL content in the network increased, but the melting temperature showed no significant changes. This confirmed, once again, the phase separation of the polymers in the network.

The fraction of PCL that crystallizes in the polymer networks was calculated applying Eq (5). The results, calculated from the first and second heating scans, are shown in Figure 8. The PCL crystalline fraction in all networks was lower than that corresponding to pure PCL. This feature is due to crosslinking between CmCHT and PCL which hinders PCL chain segment diffusion and incorporation of growing crystals. The pure PCL presented a higher crystallinity in the first than in the second scan. This is attributed to the preparation method, as PCL crystallizes from the solution during solvent casting and the corresponding crystallinity is determined in the first scan - while in the second scan PCL has been crystallized from the melt (after melting during the previous heating scan). However, the polymer CmCHT-PCL networks do not show any difference in crystallinity between the 
first and second scan. This suggests that when the PCL domains are dispersed in the CmCHT matrix, melting of the PCL crystals during the first scan produces liquid PCL micro-domains that do not diffuse in the chitosan matrix and that crystals again form on subsequent cooling - in nearly the same way as at the beginning of the measuring cycle.

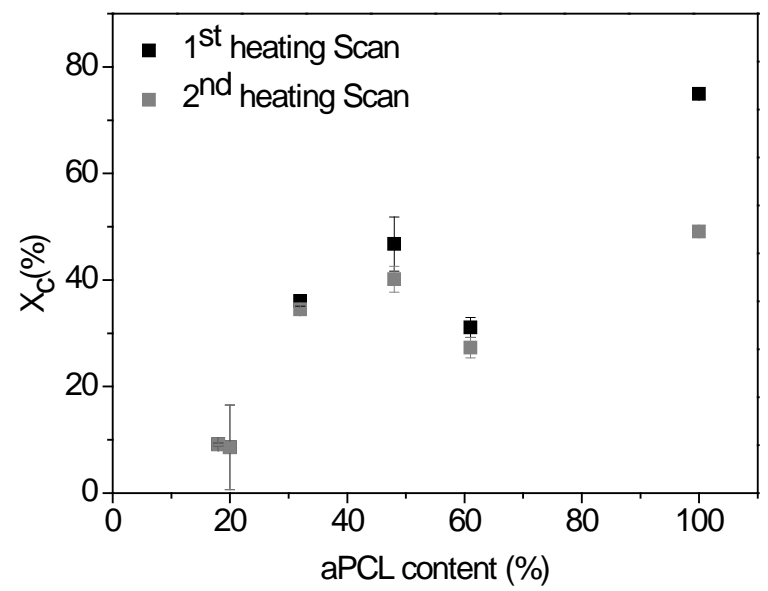

Figure 8. Crystallinity fraction of PCL as a function of PCL content in both heating scans.

The block copolymer network produced by crosslinking of CmCHT chains with aldehyde end-capped PCL is formed by two very different polymer blocks, one hydrophilic and the other hydrophobic. The polymers used to be immiscible for thermodynamic reasons [40]. The thermodynamic equilibrium criteria drive the polymer blend to phase separation. The case does not differ when the two components of the system are in the form of a network as happens in interpenetrated polymer networks [41] and even in the case of block copolymers.[42, 43] In all these cases, the formed phase domains have nanometric dimensions. The same situation is found in block copolymer networks. Polylactide-poly (hydroxyethyl acrylate) PLA-PHEA[44],[35] or PCL-PHEA [45] block copolymer networks present phase separation. Of course, chain connectivity in this kind of twocomponent polymer system can force compatibility (i.e., homogeneous miscibility of the two components at molecular level). This happens because the increasing cross-linking density of the networks leaves no space available for separated domains [46]. In the case of hydrophilic/hydrophobic systems, the immersion of the material in an aqueous medium induces phase separation - even if the dry system is homogeneous due to the phenomenon 
of hydrophobic interaction. Our results prove that phase separation takes place in a CmCHT-PCL block copolymer network. This is clearly revealed by the ability of PCL blocks to crystallize as shown both in the DSC traces of Figure 7 and by the XRD difractograms in Figure 6. It can be assumed that these PCL domains must be of nanometric dimensions due to CmCHT-PCL connectivity, and are dispersed in a continuous CmCHT phase - as proved by the water sorption capacity of the networks (around 300\% - meaning a great capacity for swelling that a network could not sustain if the hydrophobous PCL phase was continuous).

\section{Hydrolytic and enzymatic degradation tests}

The degradation profile of CmCHT-PCL polymer networks was studied under hydrolytic and enzymatic conditions. It is well known that chitosan is enzymatically degraded by lysozyme cleavage of the O-glycosidic bond, and that the PCL can be degraded by lipase [27] and in hydrolytic conditions by the cleavage of the ester groups.

PCL is a polymer susceptible to hydrolytic degradation, but the rate of mass loss when immersed in an aqueous medium is very low [47]. This is because of the high hydrophobicity and high crystallinity of this polymer. Nevertheless, hydrolytic degradation of the CmCHT-PCL networks is very fast when compared with pure PCL, and the degradation of the network must be a product of the cleavage of PCL chains since CmCHT chains are not susceptible to hydrolysis. This behavior can be explained by the dispersion of PCL in nanometric domains dispersed in water-swollen CmCHT; in this way, PCL segments in the network are readily accessible to water molecules - and so to hydrolysis. Thus $80 \mathrm{wt} \%$ of the network is degraded in just 28 days in CmCHT-93.

a)

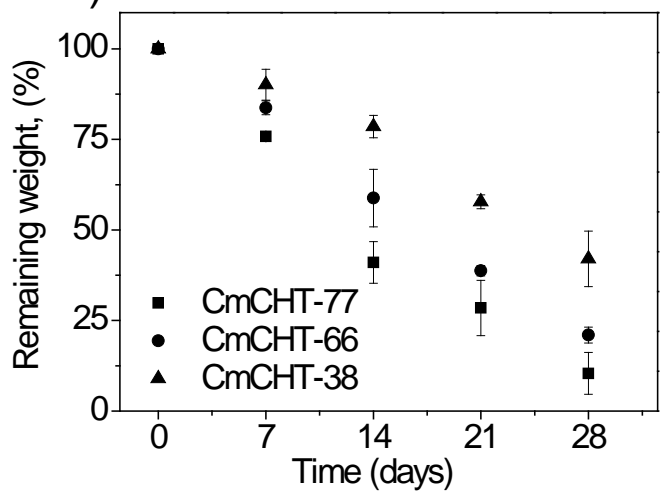

b)

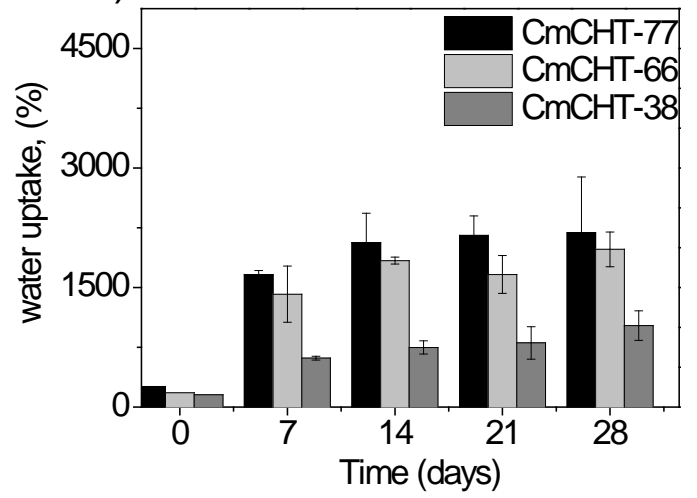


Figure 9. Hydrolytic degradation of CmCHT-PCL networks a) remaining weight, and b) water uptake. The solid lines are a guide.

When analyzing the hydrolytic degradation of chain polymers, cleavage of the polymer chains can be monitored by the decrease of the average molecular eight of the polymer and the broadening of the distribution of chin molecular weights. It is shown that molecular weight decreases significantly before the material starts losing weight. This kind of analysis is prevented in the case of polymer networks in which molecular weight tends to infinity. Nevertheless, a similar analysis can be performed by measuring the swelling capacity of the polymer network since it rapidly increases when the number of effective chains between cross-links decreases, according to Flory-Rehner theory [40] . Interestingly enough, hydrolytic degradation of CmCHT-PCL networks produces a sharp increase of equilibrium water content in the first 5 days of water immersion (Figure 9b) multiplying the water sorption capacity by a factor of 7 , while the weight loss is still quite moderated. For longer degradation times, weight loss continues while swelling capacity keeps around $2000 \%$.
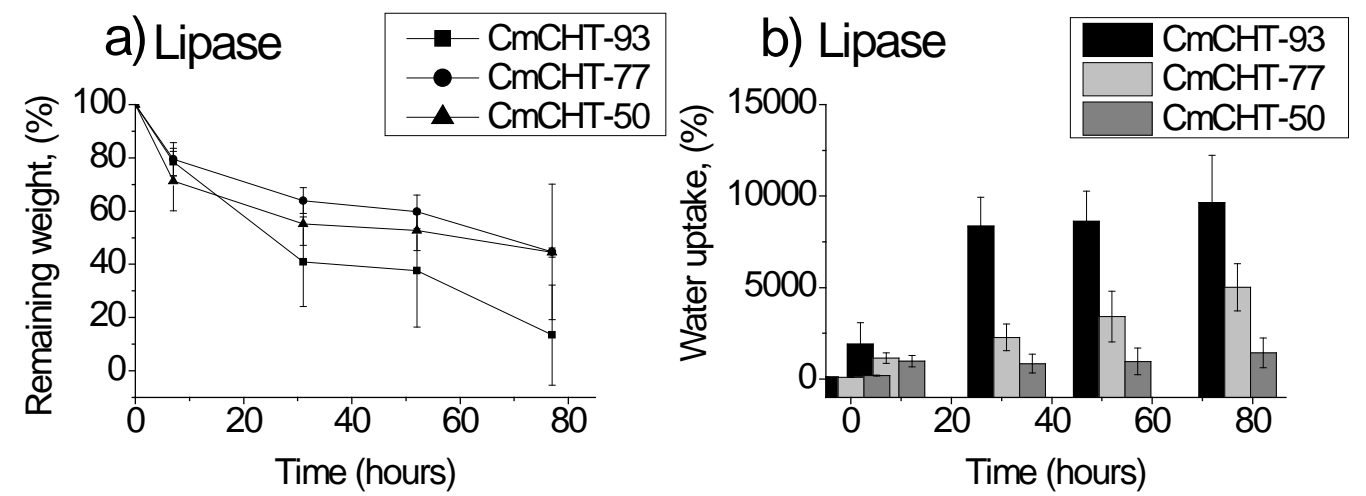

c) Lysozyme

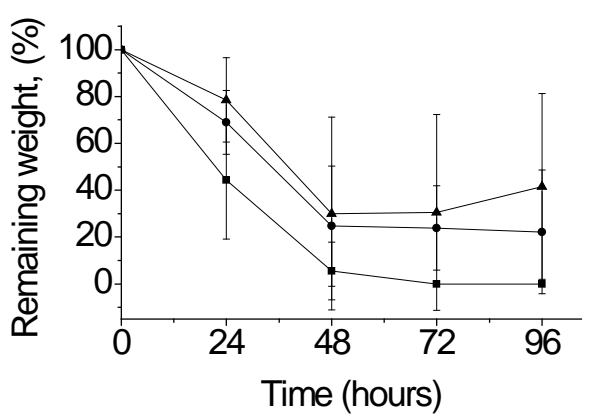

d) Lysozyme

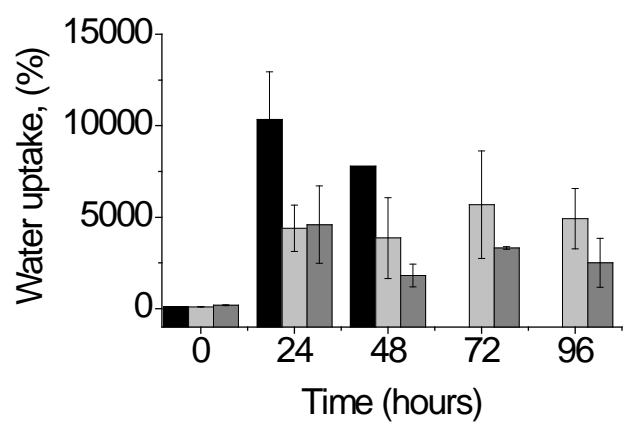

Figure 10. Evolution of sample weight and water uptake as a function of degradation process: a) and b); lipase containing medium; and c) and d) in lysozyme. The solid lines are only guides to the eye. 
Figure 10 shows the degradation profile of CmCHT-PCL networks in lipase (a) and lysozyme (c). In both cases, the samples that contain the lower amount of PCL blocks (CmCHT-93) degrade faster than the other two compositions (which show no significant differences between them). CmCHT-93 presents a remaining weight of $13 \pm 18 \%$ after 77 hours in lipase, whereas it presents no remaining weight in lysozyme. CmCHT-50 presents a similar remaining weight of $79 \pm 70 \%$ at 79 hours in lysozyme and $77 \pm 50 \%$ at 72 hours in lipase conditions. Enzymatic degradation in lipase is much faster in the case of the network than in a PCL homopolymer. Thus, while only 5wt\% of a pure PCL films were lost in 15days [38], $80 \mathrm{wt} \%$ of CmCHT-93 network is degraded in just three days.. The EWC of the degraded networks was also analyzed. The results (shown in Figure 10b and 10d) show a result similar to that of hydrolytic degradation with a sharp initial increase of the EWC both in lipase and lysozyme conditions. CmCHT-93 presented the highest EWC after degradation (as high as 1000\% after 20 hours of degradation in both enzymatic media).

To further confirm the preferential cleavage of PCL and CmCHT chains in lipase and lysozyme respectively the degraded networks were analyzed by TGA to evaluate the evolution of network composition during the enzymatic degradation process. Figure 5.10 shows the TGA profiles and the derivative for the CmCHT-50 polymer networks at different degradation times, and the pure components. The samples degraded with lipase (Figure 5.11a and 5.11b) present thermograms that evolve towards the corresponding to pristine CmCHT, indicating that the PCL component is being degraded. In the case of samples degraded in the presence of lysozyme, the TGA trace tends to become more similar to the corresponding pristine PCL as the degradation process advances, indicating that the proportion of $\mathrm{CmCHT}$ decreases with degradation time. 


\section{Lipase}
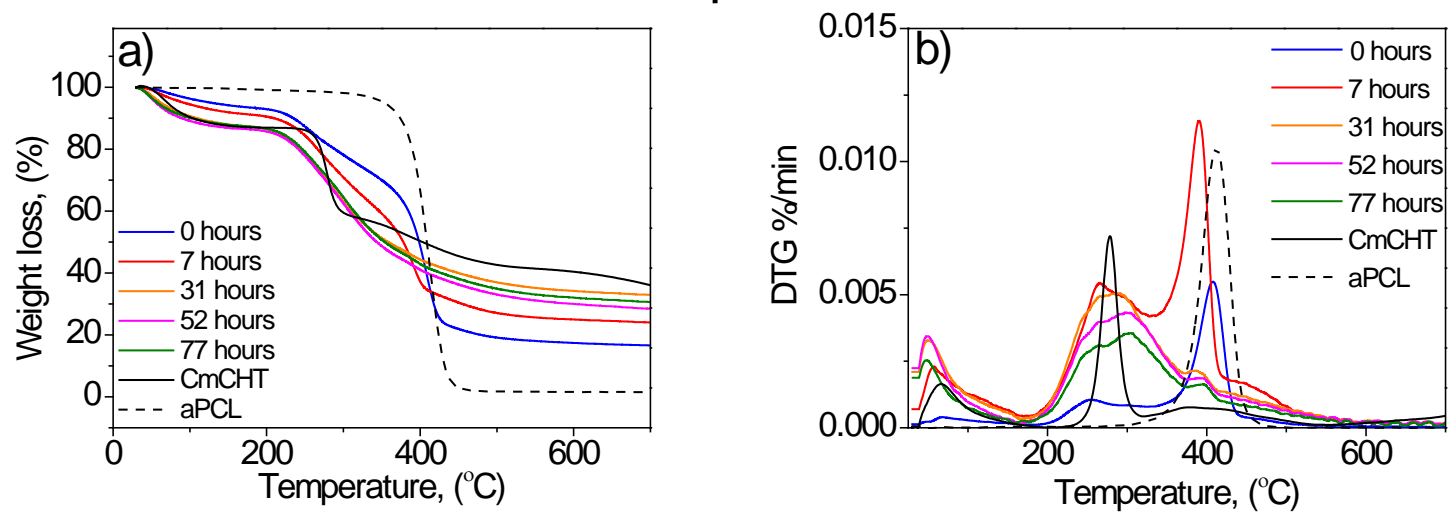

\section{Lysozyme}
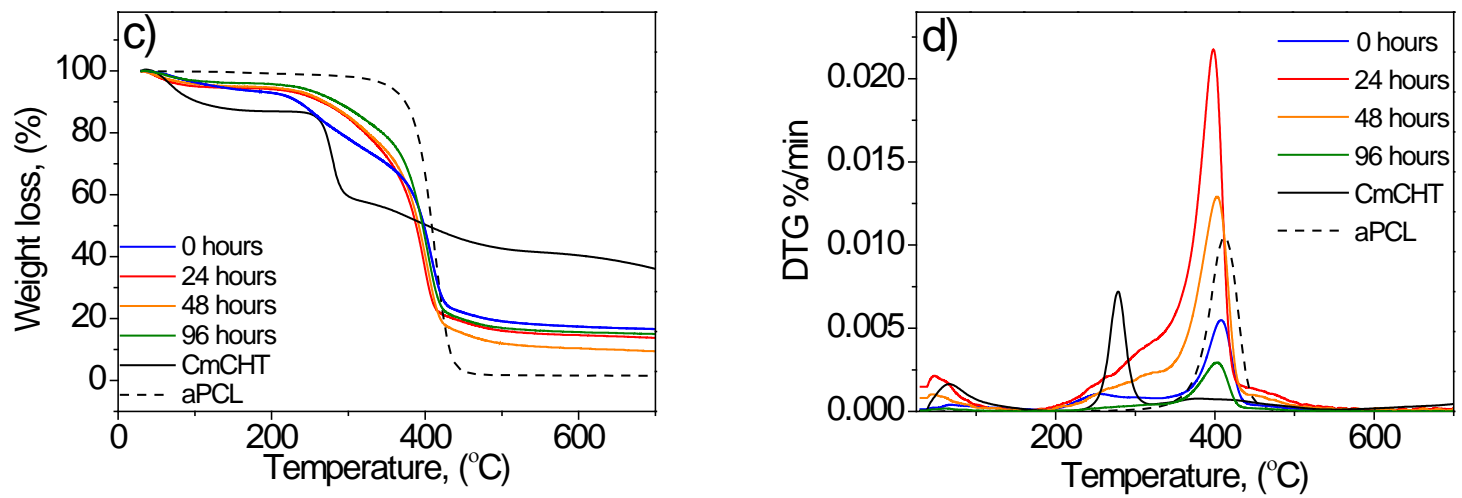

Figure 11. TGA and DTG curves from CmCHT-50 polymer network in both enzymatic media.

It is interesting to characterize the molecular weight of the chain fragments that are delivered from the network as degradation progresses in the enzymatic media. To do this, the supernatants from both enzymatic conditions were analyzed by GPC. The GPC distributions found in the case of CmCHT-50 are shown in Figure 12. Interestingly, the degradation in lipase delivers chain fragments $\left(\mathrm{Mw}=3 \times 10^{4} \mathrm{Da}, \mathrm{Mn}=2 \times 10^{4} \mathrm{Da}\right.$ at 7 hours of degradation) that are much larger than PCL blocks - but shorter than CmCHT original chains (as expected when the cleavage of PCL bridges and CmCHT chains take place). As degradation progresses larger fragments can be detected in the supernatant, but their molecular weight is always lower than that of the original CmCHT chain. Bimodal forms in the molecular weight distribution can be observed in the plots of Figure 10a: chain fragments of higher or lower molecular weight are possibly delivered from regions in the network with more or less crosslinking density. Similar results are found for the networks 
with other compositions. Nevertheless, during the degradation in lysozyme, the molecular weight of the delivered fragments does not depend significantly on the degradation time, nor on the composition of the network (with values of $\mathrm{Mw}=5 \times 10^{4} \pm 7 \times 10^{2} \mathrm{Da}, \mathrm{Mn}=2 \times 10^{4} \pm$ $5 \times 10^{2} \mathrm{Da}$ ) and single bell molecular weight distributions clearly appear (Figure 10b).
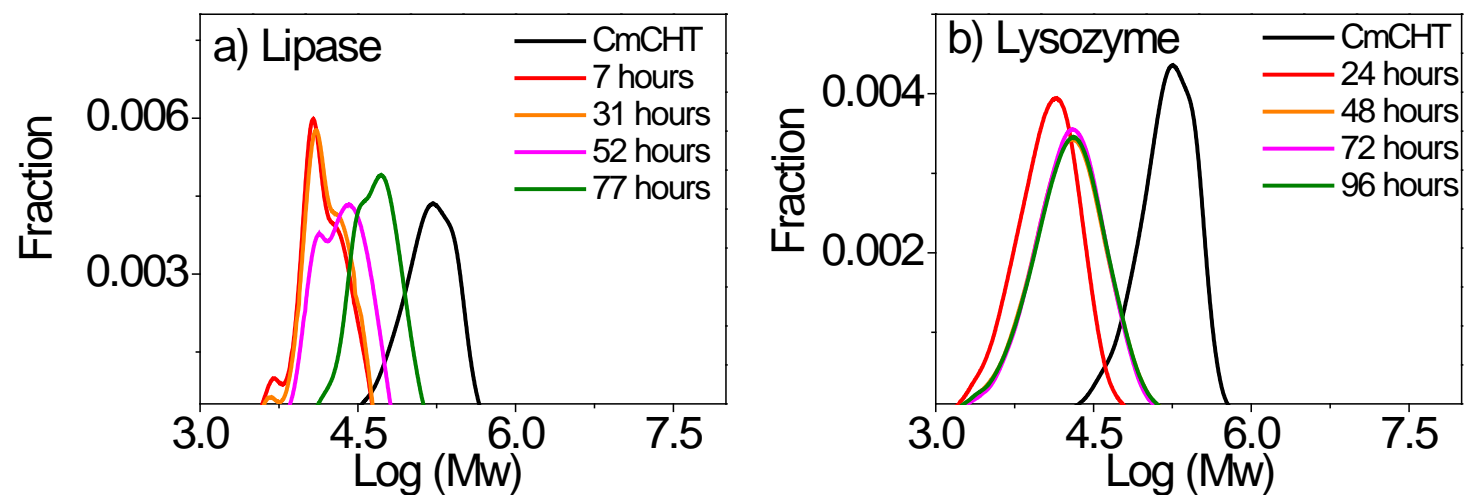

Figure 12. Changes in molecular weight distribution in supernatant solution with degradation time of CmCHT-50.

Thus, polymer chain fragments detected in the degradation medium with molecular weights of between $2 \times 10^{4}$ and $8 \times 10^{4}$ Da confirm the hypothesis that cleavage of PCL chains delivers large $\mathrm{CmCHT}$ fragments that dissolve in the aqueous medium. Lysozyme acts on CmCHT chains to produce an even quicker degradation, where the molecular weight of CmCHT fragments in the supernatant are of a similar magnitude independently of the degradation time. It seems that $\mathrm{CmCHT}$ chains cleavage by lysozyme combines with the hydrolytic degradation of the PCL blocks accelerating the degradation process.

\section{MSCs response and membrane degradation during cell culture}

Porcine MSCs were seeded into the pores of a macroporous membrane made by freeze drying, where the pore size was $51 \pm 23 \mu \mathrm{m}$ for CmCHT-50 and $117 \pm 52 \mu \mathrm{m}$ for CmCHT77. As shown in Figure 13a the membrane presents large interconnected pores. Cell seeding efficiency and cell proliferation, was analyzed by quantifying the total amount of DNA in the samples (Figure $13 \mathrm{~b}$ ). The results reveal that there are no significant differences between the cell seeding efficiency of Cm-CHT-PCL samples with varying PCL content. Total DNA values show that cell numbers increase after the first day of culture. 


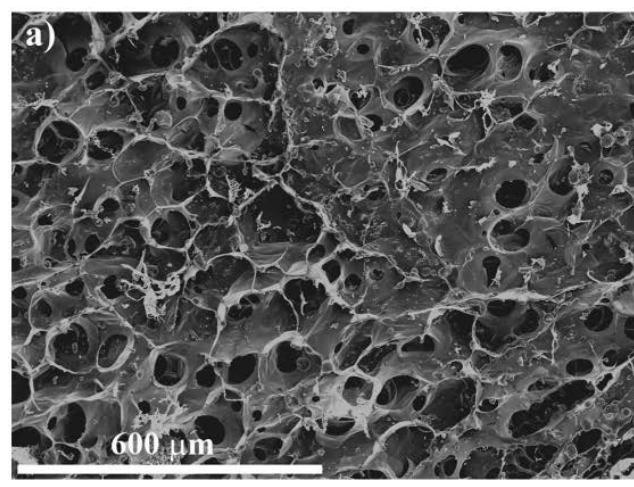

c)
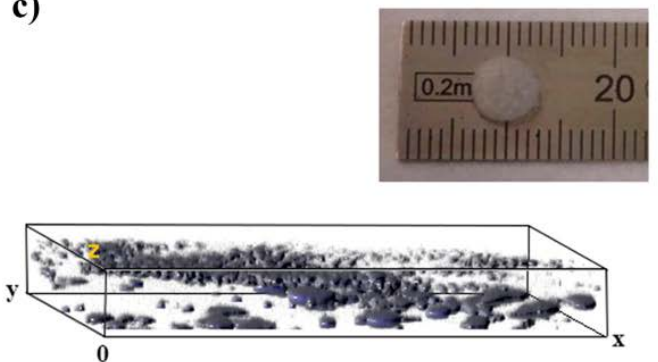

b)

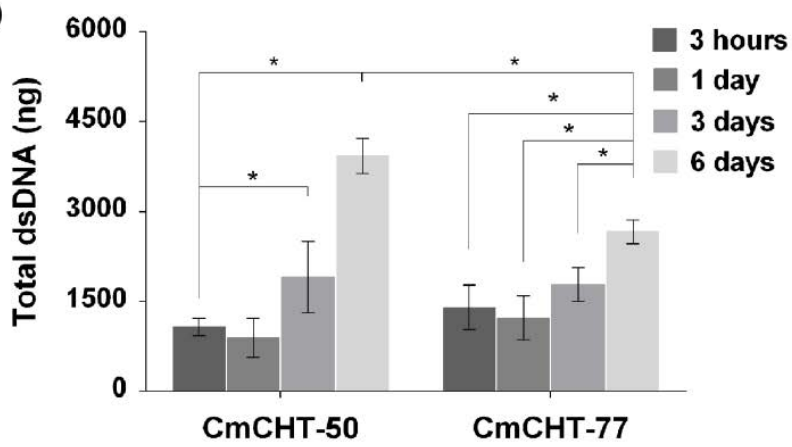

d)
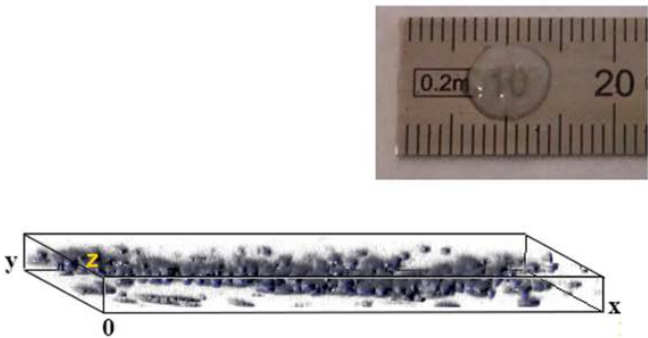

Figure 13. a) SEM micrographs of porous membranes CmCHT-50 before cell seeding, b) total dsDNA quantification of the porous membranes CmCHT-50 and CmCHT-77 for 3 hours, 1, 3 and 6 days of culture, c) and d) macroscopic view and tridimensional representation of nuclei distribution (tridimensional cell distribution) of porous membrane CmCHT-50 for 3 hours and 1 day of culture,

Confocal microscopy was used in order to analyze cells distribution inside the pores of the porous membranes. A stack of all the images taken every $10 \mu \mathrm{m}$ from the surface down to the last cell detected is analyzed. From these images, the tridimensional cell distribution (shown in figure $13 \mathrm{c}$ and d) and the value of cell penetration depth with the culture time and membranes degradation were characterized. After 3 hours of cell culture, it can be observed (Figure 13 c) that most of cells seeded on the surface penetrated into the CmCHT50 membrane $253 \pm 33 \mu \mathrm{m}$ while in CmCHT-77 they enter only up to $163 \pm 5 \mu \mathrm{m}$. This behavior can be explained by the fact that CmCHT-50 present better pore interconnectivity than CmCHT-77. Besides, as culture time increases, the membranes are degraded. Membranes of CmCHT-50 can maintain the structure and coherence until 3 days (CmCHT77 only until day one) after this time point the sample retain the shape but could not be handled (see the macroscopic view in Figure $13 \mathrm{c}$ and d), then, for longer culture times, the sample disaggregates and the culture medium becomes translucent. The content of the culture well was moved to a clean one to perform DNA analysis in order to discard the cells that could be adhered to the bottom of the well. Interestingly enough the total DNA value 
continues increasing up to 6 days culture what can be interpreted in the sense that small fragments of the membrane act as cell carriers and cells continue adhered to the biomaterial particles.

\section{Conclusions}

A new family of CmCHT-PCL networks has been synthesized using aPCL as a crosslinking agent. The main advantage of these networks compared to conventional chitosan is that they present a very fast degradation in cell culture or in enzymatic media while hydrolytic degradation takes longer but it is still much faster than in the pure components of the copolymer network. For CmCHT-77 at three days, only 50\% and 20\% of the mass is remaining in lipase and lysozyme conditions, respectively. Some $20 \%$ of the mass remains at 28 days in hydrolytic conditions. These findings are a consequence of two phenomena. Firstly, the large amount of water absorbed by the networks (despite having a hydrophobous component) facilitates the entrance of water and enzymes, and quickens PCL hydrolysis and CmCHT degradation. Secondly, as the systems are phase separated (XRD and DSC results), the domains rich in CmCHT are easily solubilized - both in enzymatic and hydrolytic conditions. Macroporous membranes made of this copolymer networks can be seeded with MSCs that are viable and proliferate inside the pores. These membranes can be used to deliver cells to the organism in regenerative therapies being resorbed in times of the order of one week. 


\section{References}

[1] M. N. V. Ravi Kumar, "A review of chitin and chitosan applications," Reactive and Functional Polymers, vol. 46, pp. 1-27, 2000.

[2] A. Di Martino, M. Sittinger, and M. V. Risbud, "Chitosan: A versatile biopolymer for orthopaedic tissue-engineering," Biomaterials, vol. 26, pp. 5983-5990, 2005.

[3] S. V. Madihally and H. W. T. Matthew, "Porous chitosan scaffolds for tissue engineering," Biomaterials, vol. 20, pp. 1133-1142, 6// 1999.

[4] M. Rodríguez-Vázquez, B. Vega-Ruiz, R. Ramos-Zúñiga, D. A. Saldaña-Koppel, and L. F. Quiñones-Olvera, "Chitosan and Its Potential Use as a Scaffold for Tissue Engineering in Regenerative Medicine," BioMed Research International, vol. 2015, 2015.

[5] M. Rinaudo, "Chitin and chitosan: properties and applications," Progress in polymer science, vol. 31, pp. 603-632, 2006.

[6] S.-i. Aiba, "Studies on chitosan: 4. Lysozymic hydrolysis of partially N-acetylated chitosans," International journal of biological macromolecules, vol. 14, pp. 225228, 1992.

[7] D. Ren, H. Yi, W. Wang, and X. Ma, "The enzymatic degradation and swelling properties of chitosan matrices with different degrees of N-acetylation," Carbohydrate Research, vol. 340, pp. 2403-2410, 2005.

[8] T. Freier, H. S. Koh, K. Kazazian, and M. S. Shoichet, "Controlling cell adhesion and degradation of chitosan films by N-acetylation," Biomaterials, vol. 26, pp. 58725878, 2005.

[9] J. Berger, M. Reist, J. M. Mayer, O. Felt, N. A. Peppas, and R. Gurny, "Structure and interactions in covalently and ionically crosslinked chitosan hydrogels for biomedical applications," European Journal of Pharmaceutics and Biopharmaceutics, vol. 57, pp. 19-34, 1// 2004.

[10] R. A. Muzzarelli, "Genipin-crosslinked chitosan hydrogels as biomedical and pharmaceutical aids," Carbohydrate Polymers, vol. 77, pp. 1-9, 2009.

[11] F.-L. Mi, H.-W. Sung, S.-S. Shyu, C.-C. Su, and C.-K. Peng, "Synthesis and characterization of biodegradable TPP/genipin co-crosslinked chitosan gel beads," Polymer, vol. 44, pp. 6521-6530, 2003.

[12] N. Kil'deeva, P. Perminov, L. Vladimirov, V. Novikov, and S. Mikhaŭlov, "[Mechanism of the reaction of glutaraldehyde with chitosan]," Bioorganicheskaia khimiia, vol. 35, pp. 397-407, 2008.

[13] E. Mirzaei B, A. Ramazani SA, M. Shafiee, and M. Danaei, "Studies on glutaraldehyde crosslinked chitosan hydrogel properties for drug delivery systems," International Journal of Polymeric Materials and Polymeric Biomaterials, vol. 62, pp. 605-611, 2013. 
[14] V. Crescenzi, A. Francescangeli, A. Taglienti, D. Capitani, and L. Mannina, "Synthesis and partial characterization of hydrogels obtained via glutaraldehyde crosslinking of acetylated chitosan and of hyaluronan derivatives," Biomacromolecules, vol. 4, pp. 1045-1054, 2003.

[15] B. Dhandayuthapani, Y. Yoshida, T. Maekawa, and D. S. Kumar, "Polymeric scaffolds in tissue engineering application: a review," International Journal of Polymer Science, vol. 2011, 2011.

[16] X. Liu, L. Ma, Z. Mao, and C. Gao, "Chitosan-based biomaterials for tissue repair and regeneration," in Chitosan for Biomaterials II, ed: Springer, 2011, pp. 81-127.

[17] A. Vidaurre, J. M. M. Dueñas, J. M. Estellés, and I. C. Cortázar, "Influence of Enzymatic Degradation on Physical Properties of Poly( $\varepsilon$-caprolactone) Films and Sponges," Macromolecular Symposia, vol. 269, pp. 38-46, 2008.

[18] J. M. Estellés, A. Vidaurre, J. M. M. Duenas, and I. C. Cortázar, "Physical characterization of polycaprolactone scaffolds," Journal of Materials Science: Materials in Medicine, vol. 19, pp. 189-195, 2008.

[19] D. M. García Cruz, J. L. Gomez Ribelles, and M. Salmerón Sánchez, "Blending polysaccharides with biodegradable polymers. I. Properties of chitosan/polycaprolactone blends," Journal of Biomedical Materials Research Part B: Applied Biomaterials, vol. 85, pp. 303-313, 2008.

[20] D. M. Garcia Cruz, D. F. Coutinho, E. Costa Martinez, J. F. Mano, J. L. Gómez Ribelles, and M. Salmerón Sánchez, "Blending polysaccharides with biodegradable polymers. II. Structure and biological response of chitosan/polycaprolactone blends," Journal of Biomedical Materials Research Part B: Applied Biomaterials, vol. 87, pp. 544-554, 2008.

[21] D. M. G. Cruz, D. F. Coutinho, J. F. Mano, J. L. G. Ribelles, and M. S. Sanchez, "Physical interactions in macroporous scaffolds based on poly ( $\varepsilon$ caprolactone)/chitosan semi-interpenetrating polymer networks," Polymer, vol. 50, pp. 2058-2064, 2009.

[22] Y. Xin and J. Yuan, "Schiff's base as a stimuli-responsive linker in polymer chemistry," Polymer Chemistry, vol. 3, pp. 3045-3055, 2012.

[23] T. Groth, M. Grøtli, W. D. Lubell, L. P. Miranda, and M. Meldal, "HYDRA: A novel hydroxy and amine functionalised resin synthesised by reductive amination of PEG aldehyde and a polyamine," Journal of the Chemical Society, Perkin Transactions 1, pp. 4258-4264, 2000.

[24] U. Shinde, M. H. Ahmed, and K. Singh, "Development of dorzolamide loaded 6-ocarboxymethyl chitosan nanoparticles for open angle glaucoma," Journal of drug delivery, vol. 2013, 2013.

[25] A. Ibn Yaich, U. Edlund, and A.-C. Albertsson, "Wood hydrolysate barriers: performance controlled via selective recovery," Biomacromolecules, vol. 13, pp. 466-473, 2012. 
[26] Crescenz.V, G. Manzini, Calzolar.G, and C. Borri, "Thermodynamics of fusion of poly-beta-propiolactone and poly-epsilon-caprolactone - comparative analysis of melting of aliphatic polylactone and polyester chains," European Polymer Journal, vol. 8, pp. 449-\&, 1972.

[27] I. Castilla-Cortázar, J. Más-Estellés, J. M. Meseguer-Dueñas, J. L. Escobar Ivirico, B. Marí, and A. Vidaurre, "Hydrolytic and enzymatic degradation of a poly $(\varepsilon-$ caprolactone) network," Polymer Degradation and Stability, vol. 97, pp. 1241$1248,8 / / 2012$.

[28] C. Antolinos-Turpín, R. M. Román, J. Rodenas-Rochina, J. G. Ribelles, and J. Gómez-Tejedor, "Macroporous thin membranes for cell transplant in regenerative medicine," Biomaterials, vol. 67, pp. 254-263, 2015.

[29] T. C. Gamboa-Martínez, J. Ródenas-Rochina, P. R. Tortosa, M. Lebourg, J. G. L. Ribelles, M. S. Sanchez, et al., "Chondrocytes cultured in an adhesive macroporous scaffold subjected to stirred flow bioreactor behave like in static culture," Journal of Biomaterials and Tissue Engineering, vol. 3, pp. 312-319, 2013.

[30] J. Ródenas-Rochina, J. L. G. Ribelles, and M. Lebourg, "Comparative study of PCL-HAp and PCL-bioglass composite scaffolds for bone tissue engineering," Journal of Materials Science: Materials in Medicine, vol. 24, pp. 1293-1308, 2013.

[31] F. R. de Abreu and S. P. Campana-Filho, "Characteristics and properties of carboxymethylchitosan," Carbohydrate Polymers, vol. 75, pp. 214-221, 1/22/ 2009.

[32] T. Wanjun, W. Cunxin, and C. Donghua, "Kinetic studies on the pyrolysis of chitin and chitosan," Polymer Degradation and Stability, vol. 87, pp. 389-394, 2005.

[33] A. V. Lluch, G. G. Ferrer, and M. M. Pradas, "Biomimetic apatite coating on P (EMA-co-HEA)/SiO 2 hybrid nanocomposites," Polymer, vol. 50, pp. 2874-2884, 2009.

[34] A. Rogina, P. Rico, G. G. Ferrer, M. Ivanković, and H. Ivanković, "In Situ Hydroxyapatite Content Affects the Cell Differentiation on Porous Chitosan/Hydroxyapatite Scaffolds," Annals of biomedical engineering, pp. 1-13, 2015.

[35] J. Escobar Ivirico, M. Salmerón-Sánchez, J. Gómez Ribelles, and M. Monleón Pradas, "Poly(l-lactide) networks with tailored water sorption," Colloid and Polymer Science, vol. 287, pp. 671-681, 2009/06/01 2009.

[36] R. J. Samuels, "Solid state characterization of the structure of chitosan films," Journal of Polymer Science: Polymer Physics Edition, vol. 19, pp. 1081-1105, 1981.

[37] M. A. Gámiz-González, A. E. Piskin, C. Pandis, C. Chatzimanolis-Moustakas, A. Kyritsis, B. Marí, et al., "Determining the influence of $\mathrm{N}$-acetylation on water sorption in chitosan films," Carbohydrate Polymers, vol. 133, pp. 110-116, 11/20/ 2015. 
[38] I. Castilla-Cortázar, J. Más-Estellés, J. M. Meseguer-Dueñas, J. E. Ivirico, B. Marí, and A. Vidaurre, "Hydrolytic and enzymatic degradation of a poly ( $\varepsilon$-caprolactone) network," Polymer Degradation and Stability, vol. 97, pp. 1241-1248, 2012.

[39] A. K. Mahanta, V. Mittal, N. Singh, D. Dash, S. Malik, M. Kumar, et al., "Polyurethane-Grafted Chitosan as New Biomaterials for Controlled Drug Delivery," Macromolecules, 2015.

[40] P. J. Flory, "Network Structure and the Elastic Properties of Vulcanized Rubber," Chemical reviews, vol. 35, pp. 51-75, 1944.

[41] E. S. Dragan, "Design and applications of interpenetrating polymer network hydrogels. A review," Chemical Engineering Journal, vol. 243, pp. 572-590, 5/1/ 2014.

[42] A. J. Campillo-Fernández, M. Salmerón Sánchez, R. Sabater i Serra, J. M. Meseguer Dueñas, M. Monleón Pradas, and J. L. Gómez Ribelles, "Water-induced (nano) organization in poly(ethyl acrylate-co-hydroxyethyl acrylate) networks," European Polymer Journal, vol. 44, pp. 1996-2004, 7// 2008.

[43] A. S. Aroca, M. M. Pradas, and J. L. Gómez Ribelles, "Macroporous poly(methyl methacrylate) produced by phase separation during polymerisation in solution," Colloid and Polymer Science, vol. 285, pp. 753-760, 2007/04/01 2007.

[44] R. Sabater i Serra, A. Kyritsis, J. Escobar Ivirico, A. Andrio Balado, J. Gómez Ribelles, P. Pissis, et al., "Structure and dynamics in poly(L-lactide) copolymer networks," Colloid and Polymer Science, vol. 288, pp. 555-565, 2010/03/01 2010.

[45] J. L. Escobar Ivirico, M. Salmerón Sánchez, R. Sabater i Serra, J. M. Meseguer Dueñas, J. L. Gómez Ribelles, and M. Monleón Pradas, "Structure and Properties of Poly(ع-caprolactone) Networks with Modulated Water Uptake," Macromolecular Chemistry and Physics, vol. 207, pp. 2195-2205, 2006.

[46] A. S. Aroca, M. M. Pradas, and J. G. Ribelles, "Effect of crosslinking on porous poly (methyl methacrylate) produced by phase separation," Colloid and Polymer Science, vol. 286, pp. 209-216, 2008.

[47] H. Tsuji, T. Ono, T. Saeki, H. Daimon, and K. Fujie, "Hydrolytic degradation of poly ( $\varepsilon$-caprolactone) in the melt," Polymer degradation and stability, vol. 89, pp. 336-343, 2005. 